\title{
Introduction to the Renormalization Group with Applications to Non-Relativistic Quantum Electron Gases
}

\author{
CIME Lectures, Cetraro \\ Vincent Rivasseau
}

May 22, 2018

\begin{abstract}
We review the rigorous work on many Fermions models which lead to the first constructions of interacting Fermi liquids in two dimensions, and allowed to prove that there are different scaling regimes in two dimensions, depending on the shape of the Fermi surface. We also review progress on the three dimensional case.

We start with a pedagogical introduction on quantum field theory and perturbative renormalization. Emphasis is then put on using renormalization around the Fermi surface in a constructive way, in which all orders of perturbation theory are summed rigorously.
\end{abstract}

\section{Introduction to QFT and Renormalization}

\subsection{Gaussian Measures}

A finite dimensional centered normalized Gaussian measure $d \mu_{C}$ is defined through its covariance. Consider a finite dimensional space $\mathbb{R}^{N}$ and a symmetric positive definite $N$ by $N$ matrix $A$. The inverse of the matrix $A$ is also a definite positive symmetric $N$ by $N$ matrix $C=A^{-1}$ called the covariance associated to $A$. The corresponding centered normalized Gaussian measure is

$$
d \mu_{C}=(2 \pi)^{-N / 2} \sqrt{\operatorname{det} A} e^{-\frac{1}{2}^{t} X A X} d^{N} X,
$$

so that $\int d \mu_{C}=1$.

To understand $d \mu_{C}$ it is better to know $C$ than $A$ since the moments or correlation functions of a Gaussian measure can be expressed simply as sums of monomials in $C$. In fact formula (1.1) perfectly makes sense if $C$ is non invertible, and even for $C=0$; but the corresponding measure has no density with respect to the Lebesgue measure in this case (for $C=0 d \mu_{C}$ is just Dirac's 
$\delta$ function at the origin). The reader familiar with eg ordinary linear PDE's knows that the essential point is to invert the matrix or the operator, hence to know the "Green's function". But quadratic forms have linear equations as their variational solutions, so both problems are linked.

Sine any function can be approximated by polynomials, probability measures are characterized by their moments, that is by the integrals they return for each polynomial of the integration variables.

The corresponding theorem which computes the moments of a Gaussian measure in terms of the covariance is fundamental in QFT and known there under the name of Wick's theorem. It expresses the result as the sum over all possible pairings or the variables of a product of covariances between the paired variables:

$$
\int X_{i_{1}} \ldots X_{i_{n}} d \mu_{C}=\sum_{G} \prod_{\ell \in G} C_{i_{b(\ell)}, i_{e(\ell)}}
$$

where $G$ runs over all Wick contractions or pairings of the labels $1, \ldots, n$. Each pair $\ell$ is pictured as a line joining two labels $b(\ell)$ and $e(\ell)$ (which we call arbitrarily the "beginning" and "end" of the line).

The theory of Gaussian measures and Wick's theorem extends to infinite dimensional spaces, in which the covariance $C$ may become a positive kernel $C(x, y)$ in a distribution space. We recall that such a kernel is an operator acting on functions through $C . f=\int C(x, y) f(y) d y$. The identity operator is represented by the Dirac kernel $C(x, y)=\delta(x-y)$. But if $C$ is positive definite with some regularity it can be also considered the covariance of a truly well defined Gaussian measure in some infinite dimensional space of distributions, through an extension of Bochner's theorem known as Minlos Theorem [1].

\section{$1.2 \quad$ Functional integrals}

In QFT, like in grand-canonical statistical mechanics, particle number is not conserved. Cross sections in scattering experiments contain the physical information of the theory ${ }^{1}$ They are the matrix elements of the diffusion matrix $\mathcal{S}$. Under suitable conditions they are expressed in terms of the Green functions $G_{N}$ of the theory through so-called "reduction formulae".

Green functions are time ordered vacuum expectation values of the field $\phi$, which is operator valued and acts on the Fock space:

$$
G_{N}\left(z_{1}, \ldots, z_{N}\right)=<\psi_{0}, T\left[\phi\left(z_{1}\right) \ldots \phi\left(z_{N}\right)\right] \psi_{0}>
$$

Here $\psi_{0}$ is the vacuum state and the $T$-product orders $\phi\left(z_{1}\right) \ldots \phi\left(z_{N}\right)$ according to increasing times.

Consider a Lagrangian field theory, and split the total Lagrangian as the sum of a free plus an interacting piece, $\mathcal{L}=\mathcal{L}_{0}+\mathcal{L}_{\text {int }}$. The Gell-Mann-Low

${ }^{1}$ Correlation functions play this fundamental role in statistical mechanics 
formula expresses the Green functions as vacuum expectation values of a similar product of free fields with an $e^{i \int \mathcal{L}_{i n t}}$ insertion:

$$
G_{N}\left(z_{1}, \ldots, z_{N}\right)=\frac{<\psi_{0}, T\left[\phi\left(z_{1}\right) \ldots \phi\left(z_{N}\right) e^{i \int d x \mathcal{L}_{i n t}(\phi(x))}\right] \psi_{0}>}{<\psi_{0}, T\left(e^{i \int d x \mathcal{L}_{i n t}(\phi(x))}\right) \psi_{0}>}
$$

In the functional integral formalism proposed by Feynman [2, the GellMann-Low formula is replaced by a functional integral in terms of an (ill-defined) "integral over histories" which is formally the product of Lebesgue measures over all space time. The corresponding formula is the Feynman-Kac formula:

$$
G_{N}\left(z_{1}, \ldots, z_{N}\right)==\frac{\int \prod_{j} \phi\left(z_{j}\right) e^{i \int \mathcal{L}(\phi(x)) d x} D \phi}{\int e^{i \int \mathcal{L}(\phi(x)) d x} D \phi} .
$$

The integrand in (1.5) contains now the full Lagrangian $\mathcal{L}=\mathcal{L}_{0}+\mathcal{L}_{\text {int }}$ instead of the interacting one. This is interesting to expose symmetries of the theory which may not be separate symmetries of the free and interacting Lagrangians, for instance gauge symmetries. Perturbation theory and the Feynman rules can still be derived as explained in the next subsection. But 1.5 is also well adapted to constrained quantization and to the study of non-perturbative effects.

For general references on QFT, see [3, 4, 5].

\subsection{Statistical Mechanics and Thermodynamic Quantities}

There is a deep analogy between the Feynman-Kac formula and the formula which expresses correlation functions in classical statistical mechanics.

The partition function of a statistical mechanics grand canonical ensemble described by a Hamiltionian $H$ at temperature $T$ and chemical potential $\mu$ is

$$
Z_{\Lambda}=\operatorname{Tr}\left(e^{-\beta(H-\mu N)}\right),
$$

where $\beta=1 / k T$, and the trace may be either a classical integration in phasespace or in the quantum case a trace on the relevant Hilbert space (Fock space).

The main problem is to compute the logarithm of the partition function. Indeed thermodynamic quantities such as eg the mean energy of the system

$$
<H>_{T, \mu}=\frac{\operatorname{Tr}\left(H e^{-\beta(H-\mu N)}\right)}{Z_{\Lambda}}=-\frac{\partial \log Z_{\lambda}}{\partial \beta},
$$

the free energy

$$
F=<H-\mu N>_{T, \mu}=-\log Z_{\Lambda},
$$

the entropy

$$
S=\frac{1}{\beta^{2}} \frac{\partial F}{\partial \beta}
$$


or the heat capacity (at fixed volume)

$$
C_{V}=\left(\frac{\partial<H>_{T, \mu}}{\partial T}\right)_{\mu}=-\frac{1}{\beta^{2}}\left(\frac{\partial<H>_{T, \mu}}{\partial \beta}\right)_{\mu}
$$

follow from that computation.

In fact all the detailed information on the equilibrium states is encoded in the list of their correlation functions, which are derivatives of the logarithm of the partition function with respect to appropriate sources. For instance for a lattice Ising model the partition function is

$$
Z_{\Lambda}=\sum_{\left\{\sigma_{x}= \pm 1\right\}} e^{-L(\sigma)}
$$

and the correlation functions are

$$
\left\langle\prod_{i=1}^{n} \sigma_{x_{i}}\right\rangle=\frac{\sum_{\left\{\sigma_{x}= \pm 1\right\}} e^{-L(\sigma)} \prod_{i} \sigma_{x_{i}}}{\sum_{\left\{\sigma_{x}= \pm 1\right\}} e^{-L(\sigma)}},
$$

where $x$ labels the discrete sites of the lattice. The sum is over configurations $\left\{\sigma_{x}= \pm 1\right\}$ which associate a "spin" with value +1 or -1 to each such site and $L(\sigma)$ contains usually nearest neighbor interactions and possibly a magnetic field h:

$$
L(\sigma)=\sum_{x, y \text { nearest neighbors }} J \sigma_{x} \sigma_{y}+\sum_{x} h \sigma_{x} .
$$

By analytically continuing 1.5 to imaginary time, or Euclidean space, it is possible to complete the analogy with 1.12 , hence to establish a firm contact between Euclidean QFT and statistical mechanics [6, 7, 8,

\subsection{Schwinger Functions}

This idea also allows to give much better meaning to the path integral, at least for a free Bosonic field. Indeed the free Euclidean measure can be defined easily as a Gaussian measure, because in Euclidean space $L_{0}$ is a quadratic form of positive typ $\AA^{2}$

The Green functions continued to Euclidean points are called the Schwinger functions of the model, and are given by the Euclidean Feynman-Kac formula:

$$
S_{N}\left(z_{1}, \ldots, z_{N}\right)=Z^{-1} \int \prod_{j=1}^{N} \phi\left(z_{j}\right) e^{-\int \mathcal{L}(\phi(x)) d x} D \phi,
$$

\footnotetext{
${ }^{2}$ However the functional space that supports this measure is not in general a space of smooth functions, but rather of distributions. This was already true for functional integrals such as those of Brownian motion, which are supported by continuous but not differentiable paths. Therefore "functional integrals" in quantum field theory should more appropriately be called "distributional integrals".
} 


$$
Z=\int e^{-\int \mathcal{L}(\phi(x)) d x} D \phi .
$$

The simplest interacting field theory is the theory of a one component scalar bosonic field $\phi$ with quartic interaction $\lambda \phi^{4}$ ( $\phi^{3}$, which is simpler, is unstable). In $\mathbb{R}^{d}$ it is called the $\phi_{d}^{4}$ model. For $d=2,3$ this model is superrenormalizable and has been built non perturbatively by constructive field theory (see [1, 9]). In these dimensions the model is unambiguously related to its perturbation series 10, 11 through Borel summability [12. For $d=4$ the model is just renormalizable, and provides the simplest pedagogical introduction to perturbative renormalization theory. But because of the Landau ghost or triviality problem explained in subsection 1.11. the model presumably does not exist as a true interacting theory at the non perturbative level (see 9 ] for a discussion of this subtle issue).

Formally the Schwinger functions of $\phi_{d}^{4}$ are the moments of the measure:

$$
d \nu=\frac{1}{Z} e^{-\frac{\lambda}{4 !} \int \phi^{4}-\left(m^{2} / 2\right) \int \phi^{2}-(a / 2) \int\left(\partial_{\mu} \phi \partial^{\mu} \phi\right)} D \phi,
$$

where

- $\lambda$ is the coupling constant, usually assumed positive or complex with positive real part; remark the arbitrary but convenient $1 / 4$ ! factor to take into account the symmetry of permutation of all fields at a local vertex.

- $m$ is the mass, which fixes an energy scale for the theory;

- $a$ is the wave function constant. It can be set to 1 by a rescaling of the field.

- $Z$ is a normalization factor which makes $(1.16)$ a probability measure;

- $D \phi$ is a formal (mathematically ill-defined) product $\prod_{x \in \mathbb{R}^{d}} d \phi(x)$ of Lebesgue measures at every point of $\mathbb{R}^{d}$.

The Gaussian part of the measure is

$$
d \mu(\phi)=\frac{1}{Z_{0}} e^{-\left(m^{2} / 2\right) \int \phi^{2}-(a / 2) \int\left(\partial_{\mu} \phi \partial^{\mu} \phi\right)} D \phi .
$$

where $Z_{0}$ is again the normalization factor which makes (1.17) a probability measure.

More precisely if we consider the translation invariant propagator $C(x, y) \equiv$ $C(x-y)$ (with slight abuse of notation), whose Fourier transform is

$$
C(p)=\frac{1}{(2 \pi)^{d}} \frac{1}{p^{2}+m^{2}},
$$

we can use Minlos theorem and the general theory of Gaussian processes to define $d \mu(\phi)$ as the centered Gaussian measure on the Schwartz space of tempered 
distributions $S^{\prime}\left(\mathbb{R}^{d}\right)$ whose covariance is $C$. A Gaussian measure is uniquely defined by its moments, or the integral of polynomials of fields. Explicitly this integral is zero for a monomial of odd degree, and for even $n=2 p$ it is equal to

$$
\int \phi\left(x_{1}\right) \ldots \phi\left(x_{n}\right) d \mu(\phi)=\sum_{\mathcal{W}} \prod_{\ell \in \mathcal{W}} C\left(x_{b(\ell)}, x_{e(\ell)}\right),
$$

where the sum runs over all the $2 p ! !=(2 p-1)(2 p-3) \ldots 5.3 .1$ Wick pairings $\mathcal{W}$ of the $2 p$ arguments into the $p$ disjoint pairs $\ell=(b(\ell), e(\ell))$.

Note that since for $d \geq 2, C(p)$ is not integrable, $C(x, y)$ must be understood as a distribution. It is therefore convenient to also use regularized kernels, for instance

$$
C_{\kappa}(p)=\frac{1}{(2 \pi)^{d}} \frac{e^{-\kappa\left(p^{2}+m^{2}\right)}}{p^{2}+m^{2}}=\int_{\kappa}^{\infty} e^{-\alpha\left(p^{2}+m^{2}\right)} d \alpha
$$

whose Fourier transform $C_{\kappa}(x, y)$ is a smooth function and not a distribution:

$$
C_{\kappa}(x, y)=\int_{\kappa}^{\infty} e^{-\alpha m^{2}-(x-y)^{2} / 4 \alpha} \frac{d \alpha}{\alpha^{D / 2}} .
$$

$\alpha^{-D / 2} e^{-(x-y)^{2} / 4 \alpha}$ is the heat kernel. Therefore this $\alpha$-representation has also an interpretation in terms of Brownian motion:

$$
C_{\kappa}(x, y)=\int_{\kappa}^{\infty} d \alpha \exp \left(-m^{2} \alpha\right) P(x, y ; \alpha)
$$

where $P(x, y ; \alpha)=(4 \pi \alpha)^{-d / 2} \exp \left(-|x-y|^{2} / 4 \alpha\right)$ is the Gaussian probability distribution of a Brownian path going from $x$ to $y$ in time $\alpha$.

Such a regulator $\kappa$ is called an ultraviolet cutoff, and we have (in the distribution sense) $\lim _{\kappa \rightarrow 0} C_{\kappa}(x, y)=C(x, y)$. Remark that due to the non zero $m^{2}$ mass term, the kernel $C_{\kappa}(x, y)$ decays exponentially at large $|x-y|$ with rate $m$. For some constant $K$ and $d>2$ we have:

$$
\left|C_{\kappa}(x, y)\right| \leq K \kappa^{1-d / 2} e^{-m|x-y|} .
$$

It is a standard useful construction to build from the Schwinger functions the connected Schwinger functions, given by:

$$
C_{N}\left(z_{1}, \ldots, z_{N}\right)=\sum_{P_{1} \cup \ldots \cup P_{k}=\{1, \ldots, N\} ; P_{i} \cap P_{j}=0}(-1)^{k+1} \prod_{i=1}^{k} S_{p_{i}}\left(z_{j_{1}}, \ldots, z_{j_{p_{i}}}\right),
$$

where the sum is performed over all distinct partitions of $\{1, \ldots, N\}$ into $k$ subsets $P_{1}, \ldots, P_{k}, P_{i}$ being made of $p_{i}$ elements called $j_{1}, \ldots, j_{p_{i}}$. For instance in the $\phi^{4}$ theory, where all odd Schwinger functions vanish due to the unbroken $\phi \rightarrow-\phi$ symmetry, the connected 4-point function is simply:

$$
\begin{aligned}
C_{4}\left(z_{1}, \ldots, z_{4}\right)= & S_{4}\left(z_{1}, \ldots, z_{4}\right)-S_{2}\left(z_{1}, z_{2}\right) S_{2}\left(z_{3}, z_{4}\right) \\
& -S_{2}\left(z_{1}, z_{3}\right) S_{2}\left(z_{2}, z_{4}\right)-S_{2}\left(z_{1}, z_{4}\right) S_{2}\left(z_{2}, z_{3}\right) .
\end{aligned}
$$





Figure 1: A possible contraction scheme with $n=N=4$.

\subsection{Feynman Graphs}

The full interacting measure may now be defined as the multiplication of the Gaussian measure $d \mu(\phi)$ by the interaction factor:

$$
d \nu=\frac{1}{Z} e^{-\frac{\lambda}{4 !} \int \phi^{4}(x) d x} d \mu(\phi)
$$

and the Schwinger functions are the normalized moments of this measure:

$$
S_{N}\left(z_{1}, \ldots, z_{N}\right)=\int \phi\left(z_{1}\right) \ldots \phi\left(z_{N}\right) d \nu(\phi) .
$$

Expanding the exponential as a power series in the coupling constant $\lambda$, one obtains a formal expansion for the Schwinger functions:

$$
S_{N}\left(z_{1}, \ldots, z_{N}\right)=\frac{1}{Z} \sum_{n=0}^{\infty} \frac{(-\lambda)^{n}}{n !} \int\left[\int \frac{\phi^{4}(x) d x}{4 !}\right]^{n} \phi\left(z_{1}\right) \ldots \phi\left(z_{N}\right) d \mu(\phi) .
$$

It is now possible to perform explicitly the functional integral of the corresponding polynomial. The result is at any order $n$ a sum over $(4 n+N-1)$ !! Wick contractions schemes $\mathcal{W}$, i.e. over all the ways of pairing together $4 n+N$ fields into $2 n+N / 2$ pairs. The weight or amplitude of such a scheme $\mathcal{W}$ is the spatial integral over $x_{1}, \ldots, x_{n}$ of the integrand $\prod_{\ell \in \mathcal{W}} C\left(x_{i_{b(\ell)}}, x_{i e(\ell)}\right)$ times the factor $\frac{1}{n !}\left(\frac{-\lambda}{4 !}\right)^{n}$. Such amplitudes are functions (in fact distributions) of the external positions $z_{1}, \ldots, z_{N}$. They may diverge either because they are integrals over all of $\mathbb{R}^{4}$ (no volume cutoff) or because the integrand is typically unbounded due to the singularities in the propagator $C$ at coinciding points.

Labeling the $n$ dummy integration variables in (1.28) as $x_{1}, \ldots, x_{n}$, we draw a line $\ell$ for each contraction of two fields. Each position $x_{1}, \ldots, x_{n}$ is then associated to a four-legged vertex and each external source $z_{i}$ to a one-legged vertex, as shown in Figure 1 .

It is convenient to draw these Wick contractions and to regroup all contractions which give rise to the same drawing or graph. There are some subtleties about labels.

Example 1.1 For the normalization at order 1 we have 4 fields, hence 3 Wick contractions, which all give the same graph. For the 2 point function at order 1 we have 6 fields, and 15 Wick contractions which fall into 2 categories with weight 3 and 12. 
We have additional observations

- The great advantage of Feynman graphs is that they form a combinatoric species in the sense of Joyal [13] whose logarithm can be computed as the species of connected graphs. As we already remarked, the computation of this logarithm is the key physical problem.

- However Feynman graphs proliferate, that is their generating functional $\sum_{n} \frac{a_{n}}{n !} \lambda^{n}$ has zero radius of convergence in $\lambda$. At the heart of any constructive strategy 11, 9, 14, 15, 16, 17, 18, lies the replacement of the proliferating species of Feynman graphs by a better one [19, typically the species of forests. The corresponding connected species is the species of trees, which does not proliferate. Indeed by Cayley's theorem there are only $n^{n-2}$ labeled trees on $n$ vertices. This is why constructive expansions converge while ordinary perturbative expansions dont. Constructive theory ultimately may be considered just as repacking Feynman graphs in some clever way according to underlying forests [20. See also the discussion in subsection 1.16 and below.

- The computation factorizes nicely into the connected components of the graphs. These components may or may not have external arguments. In the expansion for the normalized functions the vacuum components (i.e. those without external arguments) factor out and disappear. Only graphs whose connected components all contain external arguments remain.

- If we further search for elementary bricks of the expansion, we can consider the connected Schwinger functions like 1.25). In the expansion of these functions only the graphs with a single connected component containing all external arguments survive.

\subsection{Feynman Rules}

The "Feynman rules" summarize how to compute the amplitude associated to a Feynman graph with its correct combinatoric factor.

We always use the following notations for a graph $G$ :

- $n(G)$ or simply $n$ is the number of internal vertices of $G$, or the order of the graph.

- $l(G)$ or $l$ is the number of internal lines of $G$, i.e. lines hooked at both ends to an internal vertex of $G$.

- $N(G)$ or $N$ is the number of external vertices of $G$; it corresponds to the order of the Schwinger function one is looking at. When $N=0$ the graph is a vacuum graph, otherwise it is called an $N$-point graph.

- $c(G)$ or $c$ is the number of connected components of $G$,

- $L(G)$ or $L$ is the number of independent loops of G. 
For a regular $\phi^{4}$ graph, i.e. a graph which has no line hooked at both ends to external vertices, we have the relations:

$$
\begin{gathered}
l(G)=2 n(G)-N(G) / 2 \\
L(G)=l(G)-n(G)+c(G)=n(G)+1-N(G) / 2 .
\end{gathered}
$$

where in the last equality we assume connectedness of $G$, hence $c(G)=1$.

A subgraph $F$ of a graph $G$ is a subset of internal lines of $G$, together with the corresponding attached vertices. Lines in the subset defining $F$ are the internal lines of $F$, and their number is simply $l(F)$, as before. Similarly all the vertices of $G$ hooked to at least one of these internal lines of $\mathrm{F}$ are called the internal vertices of $F$ and considered to be in $F$; their number by definition is $n(F)$. Finally a good convention is to call external half-line of $F$ every half-line of $G$ which is not in $F$ but which is hooked to a vertex of $F$; it is then the number of such external half-lines which we call $N(F)$. With these conventions one has for $\phi^{4}$ subgraphs the same relation 1.29 as for regular $\phi^{4}$ graphs.

To compute the amplitude associated to a $\phi^{4}$ graph, we have to add the contributions of the corresponding contraction schemes. This is summarized by the "Feynman rules":

- To each line $\ell$ with end vertices at positions $x_{\ell}$ and $y_{\ell}$, associate a propagator $C\left(x_{\ell}, y_{\ell}\right)$.

- To each internal vertex, associate $(-\lambda) / 4$ !.

- Count all the contraction schemes giving this diagram. The number should be of the form $(4 !)^{n} n ! / S(G)$ where $S(G)$ is an integer called the symmetry factor of the diagram. The 4 ! represents the permutation of the fields hooked to an internal vertex.

- Multiply all these factors, divide by $n$ ! and sum over the position of all internal vertices.

The formula for the bare amplitude of a graph is therefore, as a distribution in $z_{1}, \ldots . z_{N}$ :

$$
A_{G}\left(z_{1}, \ldots, z_{N}\right) \equiv \int \prod_{i=1}^{n} d x_{i} \prod_{\ell \in G} C\left(x_{i_{b(\ell)}}, x_{i_{e(\ell)}}\right) .
$$

This is the "direct" or " $x$-space" representation of a Feynman integral. As stated above, this integral suffers of possible divergences. But the corresponding quantity with both volume cutoff $\Lambda$ and ultraviolet cutoff $\kappa$, namely

$$
A_{G, \Lambda}^{\kappa}\left(z_{1}, \ldots, z_{N}\right) \equiv \int_{\Lambda^{n}} \prod_{i=1}^{n} d x_{i} \prod_{\ell \in G} C_{\kappa}\left(x_{i_{b(\ell)}}, x_{i_{e(\ell)}}\right),
$$

is well defined. The integrand is indeed bounded and the integration domain $\Lambda$ is assumed compact. 
The unnormalized Schwinger functions are therefore formally given by the sum over all graphs with the right number of external lines of the corresponding Feynman amplitudes:

$$
Z S_{N}=\sum_{\phi^{4} \text { graphs } G \text { with } N(G)=N} \frac{(-\lambda)^{n(G)}}{S(G)} A_{G} .
$$

$Z$ itself, the normalization, is given by the sum of all vacuum amplitudes:

$$
Z=\sum_{\phi^{4} \text { graphs } G \text { with } N(G)=0} \frac{(-\lambda)^{n(G)}}{S(G)} A_{G} .
$$

We already remarked that the species of Feynman graphs proliferate at large orders. More precisely the total number of $\phi^{4}$ Feynman graphs at order $n$ with $N$ external arguments is $(4 n+N)$ !!. Taking into account Stirling's formula and the symmetry factor $1 / n$ ! from the exponential we expect perturbation theory at large order to behave as $K^{n} n$ ! for some constant $K$. Indeed at order $n$ the amplitude of a Feynman graph is a $4 n$-dimensional integral. It is reasonable to expect that in average it should behave as $c^{n}$ for some constant $c$. But this means that one should expect zero radius of convergence for the series (1.33). This is not too surprising. Even the one-dimensional integral

$$
F(g)=\int_{-\infty}^{+\infty} e^{-x^{2} / 2-\lambda x^{4} / 4 !} d x
$$

is well-defined only for $\lambda \geq 0$. We cannot hope infinite dimensional functional integrals of the same kind to behave better than this one dimensional integral. In mathematically precise terms, $F$ is not analytic near $\lambda=0$, but only Borel summable. Borel summability [12] is therefore the best we can hope for the $\phi^{4}$ theory, and we mentioned that it has indeed been established for the $\phi^{4}$ theory in dimensions 2 and 3 [10, 11].

From translation invariance, we do not expect $A_{G, \Lambda}^{\kappa}$ to have a limit as $\Lambda \rightarrow \infty$ if there are vacuum subgraphs in $G$. But obviously an amplitude factorizes as the product of the amplitudes of its connected components.

With simple combinatoric verification at the level of contraction schemes we can factorize the sum over all vacuum graphs in the expansion of unnormalized Schwinger functions, hence get for the normalized functions a formula analog to (1.33):

$$
S_{N}=\sum_{\substack{\phi^{4} \text { graphs } G \text { with } N(G)=N \\ G \text { without any vacuum subgraph }}} \frac{(-\lambda)^{n(G)}}{S(G)} A_{G} .
$$

Now in 1.36 it is possible to pass to the thermodynamic limit (in the sense of formal power series) because using the exponential decrease of the propagator, each individual graph has a limit at fixed external arguments. There is of course no need to divide by the volume for that because each connected component 
in 1.36 is tied to at least one external source, and they provide the necessary breaking of translation invariance.

Finally one can find the perturbative expansions for the connected Schwinger functions and the vertex functions. As expected, the connected Schwinger functions are given by sums over connected amplitudes:

$$
C_{N}=\sum_{\phi^{4} \text { connected graphs } G \text { with } N(G)=N} \frac{(-\lambda)^{n(G)}}{S(G)} A_{G}
$$

and the vertex functions are the sums of the amputated amplitudes for proper graphs, also called one-particle-irreducible. They are the graphs which remain connected even after removal of any given internal line. The amputated amplitudes are defined in momentum space by omitting the Fourier transform of the propagators of the external lines. It is therefore convenient to write these amplitudes in the so-called momentum representation:

$$
\begin{gathered}
\Gamma_{N}\left(z_{1}, \ldots, z_{N}\right)=\sum_{\phi^{4} \text { proper graphs } G \text { with } N(G)=N} \frac{(-\lambda)^{n(G)}}{S(G)} A_{G}^{T}\left(z_{1}, \ldots, z_{N}\right), \\
A_{G}^{T}\left(z_{1}, \ldots, z_{N}\right) \equiv \frac{1}{(2 \pi)^{d N / 2}} \int d p_{1} \ldots d p_{N} e^{i \sum p_{i} z_{i}} A_{G}\left(p_{1}, \ldots, p_{N}\right), \\
A_{G}\left(p_{1}, \ldots, p_{N}\right)=\int \prod_{\ell \text { internal line of } G} \frac{d^{d} p_{\ell}}{p_{\ell}^{2}+m^{2}} \prod_{v \in G} \delta\left(\sum_{\ell} \epsilon(v, \ell) p_{\ell}\right) .
\end{gathered}
$$

Remark in 1.40 the $\delta$ functions which ensure momentum conservation at each internal vertex $v$; the sum inside is over both internal and external momenta; each internal line is oriented in an arbitrary way (from $b(\ell)$ to $e(\ell)$ ) and each external line is oriented towards the inside of the graph. The incidence matrix $\epsilon(v, \ell)$ captures in a nice way the information on the internal lines 3 . It is 1 if the line $\ell$ arrives at $v,-1$ if it starts from $v$ and 0 otherwise. Remark also that there is an overall momentum conservation rule $\delta\left(p_{1}+\ldots+p_{N}\right)$ hidden in 1.40. The drawback of the momentum representation lies in the necessity for practical computations to eliminate the $\delta$ functions by a "momentum routing" prescription, and there is no canonical choice for that. Although this is rarely explicitly explained in the quantum field theory literature, such a choice of a momentum routing is equivalent to the choice of a particular spanning tree of the graph.

\subsection{Scale Analysis and Renormalization}

In order to analyze the ultraviolet or short distance limit according to the renormalization group method [22, we can cut the propagator $C$ into slices $C_{i}$ so that

\footnotetext{
${ }^{3}$ Strictly speaking this is true only for semi-regular graphs, i.e. graphs without tadpoles, i.e. without lines which start and end at the same vertex, see [21.
} 
$C=\sum_{i=0}^{\infty} C_{i}$. This can be done conveniently within the parametric representation, since $\alpha$ in this representation roughly corresponds to $1 / p^{2}$. So we can define the propagator within a slice as

$$
C_{0}=\int_{1}^{\infty} e^{-m^{2} \alpha-\frac{|x-y|^{2}}{4 \alpha}} \frac{d \alpha}{\alpha^{d / 2}}, \quad C_{i}=\int_{M^{-2 i}}^{M^{-2(i-1)}} e^{-m^{2} \alpha-\frac{|x-y|^{2}}{4 \alpha}} \frac{d \alpha}{\alpha^{d / 2}} \text { for } i \geq 1 .
$$

where $M$ is a fixed number, for instance 10 , or 2 , or $e$. We can intuitively imagine $C_{i}$ as the piece of the field oscillating with Fourier momenta essentially of size $M^{i}$. In fact it is easy to prove the bound (for $d>2$ )

$$
\left|C_{i}(x, y)\right| \leq K . M^{(d-2) i} e^{-M^{i}|x-y|}
$$

where $K$ is some constant.

Now the full propagator with ultraviolet cutoff $M^{\rho}, \rho$ being a large integer, may be viewed as a sum of slices:

$$
C_{\leq \rho}=\sum_{i=0}^{\rho} C_{i}
$$

Then the basic renormalization group step is made of two main operations:

- A functional integration

- The computation of a logarithm

Indeed decomposing a covariance in a Gaussian process corresponds to a decomposition of the field into independent Gaussian random variables $\phi^{i}$, each distributed with a measure $d \mu_{i}$ of covariance $C_{i}$. Let us introduce

$$
\Phi_{i}=\sum_{j=0}^{i} \phi_{j}
$$

This is the "low-momentum" field for all frequencies lower than $i$. The RG idea is that starting from scale $\rho$ and performing $\rho-i$ steps, one arrives at an effective action for the remaining field $\Phi_{i}$. Then, writing $\Phi_{i}=\phi_{i}+\Phi_{i-1}$, one splits the field into a "fluctuation" field $\phi_{i}$ and a "background" field $\Phi_{i-1}$. The first step, functional integration, is performed solely on the fluctuation field, so it computes

$$
Z_{i-1}\left(\Phi_{i-1}\right)=\int d \mu_{i}\left(\phi_{i}\right) e^{-S_{i}\left(\phi_{i}+\Phi_{i-1}\right)}
$$

Then the second step rewrites this quantity as the exponential of an effective action, hence simply computes

$$
S_{i-1}\left(\Phi_{i-1}\right)=-\log \left[Z_{i-1}\left(\Phi_{i-1}\right)\right]
$$

Now $Z_{i-1}=e^{-S_{i-1}}$ and one can iterate! The flow from the initial bare action $S=S_{\rho}$ for the full field to an effective renormalized action $S_{0}$ for the last "slowly 



Figure 2: A high energy subgraph $\mathbf{S}$ seen from lower energies looks quasi-local.

varying" component $\phi_{0}$ of the field is similar to the flow of a dynamical system. Its evolution is decomposed into a sequence of discrete steps from $S_{i}$ to $S_{i-1}$.

This renormalization group strategy can be best understood on the system of Feynman graphs which represent the perturbative expansion of the theory. The first step, functional integration over fluctuation fields, means that we have to consider subgraphs with all their internal lines in higher slices than any of their external lines. The second step, taking the logarithm, means that we have to consider only connected such subgraphs. We call such connected subgraphs quasi-local. Renormalizability is then a non trivial result that combines locality and power counting for these quasi-local subgraphs.

\subsection{Locality, Power Counting}

Locality simply means that quasi-local subgraphs $S$ look local when seen through their external lines. Indeed since they are connected and since their internal lines have scale say $\geq i$, all the internal vertices are roughly at distance $M^{-i}$. But the external lines have scales $\leq i-1$, which only distinguish details larger than $M^{-(i-1)}$. Therefore they cannot distinguish the internal vertices of $S$ one from the other. Hence quasi-local subgraphs look like "fat dots" when seen through their external lines, see Figure 2 Obviously this locality principle is completely independent of dimension.

Power counting is a rough estimate which compares the size of a fat dot such as $S$ in Figure 2 with $N$ external legs to the coupling constant that would be in front of an exactly local $\int \phi^{N}(x) d x$ interaction term if it were in the Lagrangian. To simplify we now assume that the internal scales are all equal to $i$, the external scales are $O(1)$, and we do not care about constants and so on, but only about the dependence in $i$ as $i$ gets large. We must first save one internal position such as the barycenter of the fat dot or the position of a particular internal vertex to represent the $\int d x$ integration in $\int \phi^{N}(x) d x$. Then we must integrate over the positions of all internal vertices of the subgraph save that one. This brings about a weight $M^{-d i(n-1)}$, because since $S$ is connected we can use the decay of the internal lines to evaluate these $n-1$ integrals. Finally we should not forget the prefactor $M^{(D-2) l i}$ coming from 1.42 , for the 
$l$ internal lines. Multiplying these two factors and using relation $(1.29)-(1.30)$ we obtain that the "coupling constant" or factor in front of the fat dot is of order $M^{-d i(n-1)+2 i(2 n-N / 2)}=M^{\omega(G)}$, if we define the superficial degree of divergence of a $\phi_{d}^{4}$ connected graph as:

$$
\omega(G)=(d-4) n(G)+d-\frac{d-2}{2} N(G) .
$$

So power counting, in contrast with locality, depends on the space-time dimension.

Let us return to the concrete example of Figure 2, A 4-point subgraph made of three vertices and four internal lines at a high slice $i$ index. If we suppose the four external dashed lines have much lower index, say of order unity, the subgraph looks almost local, like a fat dot at this unit scale. We have to save one vertex integration for the position of the fat dot. Hence the coupling constant of this fat dot is made of two vertex integrations and the four weights of the internal lines (in order not to forget these internal line factors we kept internal lines apparent as four tadpoles attached to the fat dot in the right of Figure 2). In dimension 4 this total weight turns out to be independent of the scale.

\subsection{Renormalization, Effective Constants}

At lower scales propagators can branch either through the initial bare coupling or through any such fat dot in all possible ways because of the combinatorial rules of functional integration. Hence they feel effectively a new coupling which is the sum of the bare coupling plus all the fat dot corrections coming from higher scales. To compute these new couplings only graphs with $\omega(G) \geq 0$, which are called primitively divergent, really matter because their weight does not decrease as the gap $i$ increases.

- If $d=2$, we find $\omega(G)=2-2 n$, so the only primitively divergent graphs have $n=1$, and $N=0$ or $N=2$. The only divergence is due to the "tadpole" loop $\int \frac{d^{2} p}{\left(p^{2}+m^{2}\right)}$ which is logarithmically divergent.

- If $d=3$, we find $\omega(G)=3-n-N / 2$, so the only primitively divergent graphs have $n \leq 3, N=0$, or $n \leq 2$ and $N=2$. Such a theory with only a finite number of "primitively divergent" subgraphs is called superrenormalizable.

- If $d=4, \omega(G)=4-N$. Every two point graph is quadratically divergent and every four point graph is logarithmically divergent. This is in agreement with the superficial degree of these graphs being respectively 2 and 0 . The couplings that do not decay with $i$ all correspond to terms that were already present in the Lagrangian, namely $\int \phi^{4}, \int \phi^{2}$ and $\int(\nabla \phi) \cdot(\nabla \phi)^{4}$. Hence the structure of the Lagrangian resists under change of scale, although the values of the coefficients can change. The theory is called just renormalizable.

\footnotetext{
${ }^{4}$ Because the graphs with $N=2$ are quadratically divergent we must Taylor expand the quasi local fat dots until we get convergent effects. Using parity and rotational symmetry, this generates only a logarithmically divergent $\int(\nabla \phi) \cdot(\nabla \phi)$ term beyond the quadratically divergent $\int \phi^{2}$. Furthermore this term starts only at $n=2$ or two loops, because the first tadpole graph at $N=2, n=1$ is exactly local.
} 
- Finally for $d>4$ we have infinitely many primitively divergent graphs with arbitrarily large number of external legs, and the theory is called nonrenormalizable, because fat dots with $N$ larger than 4 are important and they correspond to new couplings generated by the renormalization group which are not present in the initial bare Lagrangian.

To summarize:

- Locality means that quasi-local subgraphs look local when seen through their external lines. It holds in any dimension.

- Power counting gives the rough size of the new couplings associated to these subgraphs as a function of their number $N$ of external legs, of their order $n$ and of the dimension of space time $d$.

- Renormalizability (in the ultraviolet regime) holds if the structure of the Lagrangian resists under change of scale, although the values of the coefficients or coupling constants may change. For $\phi^{4}$ it occurs if $d \leq 4$, with $d=4$ the most interesting case.

\subsection{The BPHZ Theorem}

The BPHZ theorem is both a brilliant historic piece of mathematical physics which gives precise mathematical meaning to the notion of renormalizability, using the mathematics of formal power series, but it is also ultimately a dead end and a bad way to understand and express renormalization. Let us try to explain both statements.

For the massive Euclidean $\phi_{4}^{4}$ theory we could for instance state the following normalization conditions on the connected functions in momentum space at zero momenta:

$$
\begin{aligned}
C^{4}(0,0,0,0) & =-\lambda_{r e n}, \\
C^{2}\left(p^{2}=0\right) & =\frac{1}{m_{r e n}^{2}}, \\
\left.\frac{d}{d p^{2}} C^{2}\right|_{p^{2}=0} & =-\frac{a_{r e n}}{m_{r e n}^{4}} .
\end{aligned}
$$

Usually one puts $a_{r e n}=1$ by rescaling the field $\phi$.

Using the inversion theorem on formal power series for any fixed ultraviolet cutoff $\kappa$ it is possible to reexpress any formal power series in $\lambda_{\text {bare }}$ with bare propagators $1 /\left(a_{\text {bare }} p^{2}+m_{\text {bare }}^{2}\right)$ for any Schwinger functions as a formal power series in $\lambda_{\text {ren }}$ with renormalized propagators $1 /\left(a_{r e n} p^{2}+m_{r e n}^{2}\right)$. The BPHZ theorem then states that that formal perturbative formal power series has finite coefficients order by order when the ultraviolet cutoff $\kappa$ is lifted. The first proof by Hepp [25] relied on the inductive Bogoliubov's recursion scheme [?]. Then a completely explicit expression for the coefficients of the renormalized series was written by Zimmermann and many followers [27. The coefficients of that renormalized series can ne written as sums of renormalized Feynman amplitudes. They are similar to Feynman integrals but with additional subtractions 
indexed by Zimmermann's forests. Returning to an inductive rather than explicit scheme, Polchinski remarked that it is possible to also deduce the BPHZ theorem from a renormalization group equation and inductive bounds which does not decompose each order of perturbation theory into Feynman graphs [28. This method was clarified and applied by C. Kopper and coworkers, see [?].

The solution of the difficult "overlapping" divergence problem through Bogoliubov's or Polchinski's recursions and Zimmermann's forests becomes particularly clear in the parametric representation using Hepp's sectors. A Hepp sector is simply a complete ordering of the $\alpha$ parameters for all the lines of the graph. In each sector there is a different classification of forests into packets so that each packet gives a finite integral [30, 31, 32.

But from the physical point of view we cannot conceal the fact that purely perturbative renormalization theory is not very satisfying. At least two facts hint at a better theory which lies behind:

- The forest formula seems unnecessarily complicated, with too many terms. For instance in any given Hepp sector only one particular packet of forests is really necessary to make the renormalized amplitude finite, the one which corresponds to the quasi-local divergent subgraphs of that sector. The other packets seem useless, a little bit like "junk DNA". They are there just because they are necessary for other sectors. This does not look optimal.

- The theory makes renormalized amplitudes finite, but at tremendous cost! The size of some of these renormalized amplitudes becomes unreasonably large as the size of the graph increases. This phenomenon is called the "renormalon problem". For instance it is easy to check that the renormalized amplitude (at 0 external momenta) of the graphs $P_{n}$ with 6 external legs and $n+2$ internal vertices in Figure 3 becomes as large as $c^{n} n$ ! when $n \rightarrow \infty$. Indeed at large $q$ the renormalized amplitude $A_{G_{2}}^{R}$ in Figure 5 grows like $\log |q|$. Therefore the chain of $n$ such graphs in Figure 3 behaves as $[\log |q|]^{n}$, and the total amplitude of $P_{n}$ behaves as

$$
\int[\log |q|]^{n} \frac{d^{4} q}{\left[q^{2}+m^{2}\right]^{3}} \simeq_{n \rightarrow \infty} c^{n} n !
$$

So after renormalization some families of graphs acquire so large values that they cannot be resummed! Physically this is just as bad as if infinities were still there.

These two hints are in fact linked. As their name indicates, renormalons are due to renormalization. Families of completely convergent graphs such as the graphs $Q_{n}$ of Figure 4, are bounded by $c^{n}$, and produce no renormalons.

Studying more carefully renormalization in the $\alpha$ parametric representation one can check that renormalons are solely due to the forests packets that we compared to "junk DNA". Renormalons are due to subtractions that are not 


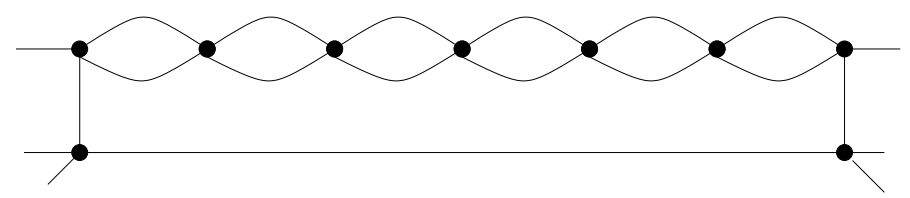

Figure 3: A family of graphs $P_{n}$ producing a renormalon.

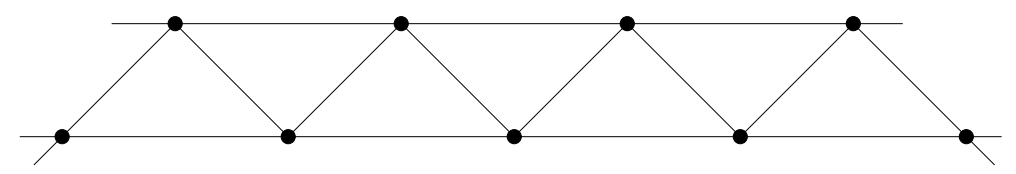

Figure 4: A family of convergent graphs $Q_{n}$, that do not produce any renormalon.

necessary to ensure convergence, just like the strange $\log |q|$ growth of $A_{G_{0}}^{R}$ at large $q$ is solely due to the counterterm in the region where this counterterm is not necessary to make the amplitude finite.

We can therefore conclude that subtractions are not organized in an optimal way by the Bogoliubov recursion. What is wrong from a physical point of view in the BPHZ theorem is to use the size of the graph as the relevant parameter to organize Bogoliubov's induction. It is rather the size of the line momenta that should be used to better organize the renormalization subtractions.

This leads to the point of view advocated in 9]: neither the bare nor the renormalized series are optimal. Perturbation should be organized as a power series in an infinite set of effective expansions, which are related through the RG flow equation. In the end exactly the same contributions are resummed than in the bare or in the renormalized series, but they are regrouped in a much better way.

\subsection{The Landau ghost and Asymptotic Freedom}

In the case of $\phi_{4}^{4}$ only the flow of the coupling constants really matters, because the flow of $m$ and of $a$ for different reasons are not very important in the ultraviolet limit:

- the flow of $m$ is governed at leading order by the tadpole. The bare mass $m_{i}^{2}$ corresponding to a finite positive physical mass $m_{r e n}^{2}$ is negative and grows as $\lambda M^{2 i}$ with the slice index $i$. But since $p^{2}$ in the $i$-th slice is also of order $M^{2 i}$ but without the $\lambda$, as long as the coupling $\lambda$ remains small it remains much larger than $m_{i}^{2}$. Hence the mass term plays no significant role in the higher slices. It was remarked in [9] that because there are no overlapping problem associated to 1PI two point subgraphs, there is in fact no inconvenience to use the full renormalized $m_{\text {ren }}$ all the way from the bare to renormalized scales, with subtractions on 1PI two point subgraphs independent of their scale. 

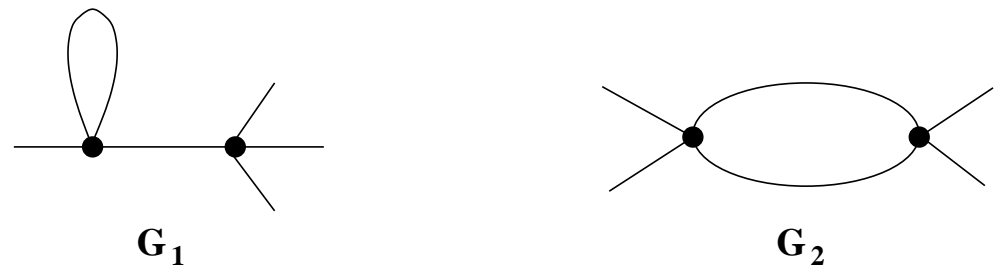

Figure 5: The $\phi^{4}$ connected graphs with $n=2, N=4$.

- the flow of $a$ is also not very important. Indeed it really starts at two loops because the tadpole is exactly local. So this flow is in fact bounded, and generates no renormalons. In fact as again remarked in [9] for theories of the $\phi_{4}^{4}$ type one might as well use the bare value $a_{\text {bare }}$ all the way from bare to renormalized scales and perform no second Taylor subtraction on any 1PI two point subgraphs.

But the physics of $\phi_{4}^{4}$ in the ultraviolet limit really depends of the flow of $\lambda$. By a simple second order computation there are only 2 connected graphs with $n=2$ and $N=4$ pictured in Figure 5 . They govern at leading order the flow of the coupling constant.

In the commutative $\phi_{4}^{4}$ theory the graph $G_{1}$ does not contribute to the coupling constant flow. This can be seen in many ways, for instance after mass renormalization the graph $G_{1}$ vanishes exactly because it contains a tadpole which is not quasi-local but exactly local. One can also remark that the graph is one particle reducible. In ordinary translation-invariant, hence momentumconserving theories, one-particle-reducible quasi-local graphs never contribute significantly to RG flows. Indeed they become very small when the gap $i$ between internal and external scales grows. This is because by momentum conservation the momentum of any one-particle-reducible line $\ell$ has to be the sum of a finite set of external momenta on one of its sides. But a finite sum of small momenta remains small and this clashes directly with the fact that $\ell$ being internal its momentum should grow as the gap $i$ grows. Remark that this is no longer be true in non commutative vulcanized $\phi_{4}^{\star 4}$, because that theory is not translation invariant, and that's why it will ultimately escape the Landau ghost curse.

So in $\phi_{4}^{4}$ the flow is intimately linked to the sign of the graph $G_{2}$ of Figure 5 . More precisely, we find that at second order the relation between $\lambda_{i}$ and $\lambda_{i-1}$ is

$$
\lambda_{i-1} \simeq \lambda_{i}-\beta \lambda_{i}^{2}
$$

(remember the minus sign in the exponential of the action), where $\beta$ is a constant, namely the asymptotic value of $\sum_{j, j^{\prime} / \inf \left(j, j^{\prime}\right)=i} \int d^{4} y C_{j}(x, y) C_{j^{\prime}}(x, y)$ when $i \rightarrow \infty$. Clearly this constant is positive. So for the normal stable $\phi_{4}^{4}$ theory, the relation 1.52 inverts into

$$
\lambda_{i} \simeq \lambda_{i-1}+\beta \lambda_{i-1}^{2},
$$

so that fixing the renormalized coupling seems to lead at finite $i$ to a large, diverging bare coupling, incompatible with perturbation theory. This is the 
Landau ghost problem, which affects both the $\phi_{4}^{4}$ theory and electrodynamics. Equivalently if one keeps $\lambda_{i}$ finite as $i$ gets large, $\lambda_{0}=\lambda_{\text {ren }}$ tends to zero and the final effective theory is "trivial" which means it is a free theory without interaction, in contradiction with the physical observation e.g. of a coupling constant of about $1 / 137$ in electrodynamics.

But in non-Abelian gauge theories an extra minus sign is created by the algebra of the Lie brackets. This surprising discovery has deep consequences. The flow relation becomes approximately

$$
\lambda_{i} \simeq \lambda_{i-1}-\beta \lambda_{i} \lambda_{i-1},
$$

with $\beta>0$, or, dividing by $\lambda_{i} \lambda_{i-1}$,

$$
1 / \lambda_{i} \simeq 1 / \lambda_{i-1}+\beta,
$$

with solution $\lambda_{i} \simeq \frac{\lambda_{0}}{1+\lambda_{0} \beta i}$. A more precise computation to third order in fact leads to

$$
\lambda_{i} \simeq \frac{\lambda_{0}}{1+\lambda_{0}(\beta i+\gamma \log i+O(1))} .
$$

Such a theory is called asymptotically free (in the ultraviolet limit) because the effective coupling tends to 0 with the cutoff for a finite fixed small renormalized coupling. Physically the interaction is turned off at small distances. This theory is in agreement with scattering experiments which see a collection of almost free particles (quarks and gluons) inside the hadrons at very high energy. This was the main initial argument to adopt quantum chromodynamics, a non-Abelian gauge theory with $S U(3)$ gauge group, as the theory of strong interactions [?].

Remark that in such asymptotically free theories which form the backbone of today's standard model, the running coupling constants remain bounded between far ultraviolet "bare" scales and the lower energy scale where renormalized couplings are measured. Ironically the point of view on early renormalization theory as a trick to hide the ultraviolet divergences of QFT into infinite unobservable bare parameters could not turn out to be more wrong than in the standard model. Indeed the bare coupling constants tend to 0 with the ultraviolet cutoff, and what can be farther from infinity than 0 ?

Recently it has been shown to all orders of perturbation theory that there should be no Landau ghost but an asymptotically safe fixed point for the similar RG flow of the non-commutative Grosse-Wulkenhaar $\phi_{4}^{\star 4}$ model [34, 35, 36, 37. Therefore this model is a kind of "Ising model" for just renormalizable QFT, that is a simple model in which one can presumably fully mathematically control at last the phenomenon of renormalization in 11 its aspects.

\subsection{Grassmann representations of determinants and Pfaf- fians}

Independent Grassmann variables $\chi_{1}, \ldots, \chi_{n}$ satisfy complete anticommutation relations

$$
\chi_{i} \chi_{j}=-\chi_{j} \chi_{i} \quad \forall i, j
$$


so that any function of these variables is a polynomial with highest degree one in each variable.

The rules of Grassmann integration are defined by linearity and

$$
\int d \chi_{i}=0, \quad \int \chi_{i} d \chi_{i}=1 .
$$

plus the rule that all $d \chi$ symbols also anticommute between themselves and with all $\chi$ variables.

The main important facts are

- Any function of Grassmann variables is a polynomial with highest degree one in each variable.

- Pfaffians and determinants can be nicely written as Grassmann integrals;

The determinant of any $n$ by $n$ matrix can indeed be expressed as a Grassmann Gaussian integral over $2 n$ independent Grassmann variables which it is convenient to name as $\bar{\psi}_{1}, \ldots, \bar{\psi}_{n}, \psi_{1}, \ldots, \psi_{n}$, although the bars have nothing yet at this stage to do with complex conjugation. The formula is

$$
\operatorname{det} M=\int \prod d \bar{\psi}_{i} d \psi_{i} e^{-\sum_{i j} \bar{\psi}_{i} M_{i j} \psi_{j}} .
$$

Remember that for ordinary commuting variables and a positive $n$ by $n$ Hermitian matrix $M$

$$
\frac{1}{\pi^{n}} \int_{-\infty}^{+\infty} \prod_{i} d \bar{\phi}_{i} d \phi_{i} e^{-\sum_{i j} \bar{\phi}_{i} M_{i j} \phi_{j}}=\frac{1}{\operatorname{det} M} .
$$

In short Grassmann Gaussian measures are simpler than ordinary Gaussian measures for two main reasons:

- Grassmann Gaussian measures are associated to any matrix $M$, there is no positivity requirement for $M$ like for ordinary Gaussian measures.

- Their normalization directly computes the determinant of $M$, not the inverse (square-root of) the determinant of $M$. This is essential in many areas where factoring out this determinant is desirable; it explains in particular the success of Grassmann and supersymmetric functional integrals in the study of disordered systems.

The stubborn reader which remembers the square-root formula in 1.1 and would like to understand the corresponding "real version" of $(1.58)$ is rewarded by the beautiful theory of Pfaffians. Clearly commuting Gaussian real integrals involve symmetric matrices, but Grassmann Gaussian with only $n$ "real" integrals must involve $n$ by $n$ antisymmetric matrices.

The $\operatorname{Pfaffian} \operatorname{Pf}(A)$ of an antisymmetric matrix $A$ is defined by

$$
\operatorname{det} A=[\operatorname{Pf}(A)]^{2} .
$$


and is known to be polynomila in the coefficients of $A$. This fact is recovered easily by writing it as

$$
\operatorname{Pf}(A)=\int d \chi_{1} \ldots d \chi_{n} e^{-\sum_{i<j} \chi_{i} A_{i j} \chi_{j}}=\int d \chi_{1} \ldots d \chi_{n} e^{-\frac{1}{2} \sum_{i, j} \chi_{i} A_{i j} \chi_{j}} .
$$

Indeed we have

$$
\operatorname{det} A=\int \prod_{i} d \bar{\psi}_{i} d \psi_{i} e^{-\sum_{i j} \bar{\psi}_{i} A_{i j} \psi_{j}} .
$$

Performing the change of variables (which a posteriori justifies the complex notation)

$$
\bar{\psi}_{i}=\frac{1}{\sqrt{2}}\left(\chi_{i}-i \omega_{i}\right), \quad \psi_{i}=\frac{1}{\sqrt{2}}\left(\chi_{i}+i \omega_{i}\right)
$$

whose Jacobian is $i^{-n}$, the new variables $\chi$ and $\omega$ are again independent Grassmann variables. Now a short computation using $A_{i j}=-A_{j i}$ gives

$$
\begin{aligned}
\operatorname{det} A & =i^{-n} \int \prod_{i} d \chi_{i} d \omega_{i} e^{-\sum_{i<j} \chi_{i} A_{i j} \chi_{j}-\sum_{i<j} \omega_{i} A_{i j} \omega_{j}} \\
& =\int \prod_{i} d \chi_{i} e^{-\sum_{i<j} \chi_{i} A_{i j} \chi_{j}} \prod_{i} d \omega_{i} e^{-\sum_{i<j} \omega_{i} A_{i j} \omega_{j}},
\end{aligned}
$$

where we used that $n=2 p$ has to be even and that a factor $(-1)^{p}$ is generated when changing $\prod_{i} d \chi_{i} d \omega_{i}$ into $\prod_{i} d \chi_{i} \prod_{i} d \omega_{i}$. Equation (1.64) shows why $\operatorname{det} A$ is a perfect square and proves 1.61.

A useful Lemma is:

Lemma 1.2 The determinant of a matrix $D+A$ where $D$ is diagonal and $A$ antisymmetric has a "quasi-Pfaffian" representation

$$
\operatorname{det}(D+A)=\int \prod_{i} d \chi_{i} d \omega_{i} e^{-\sum_{i} \chi_{i} D_{i i} \omega_{i}-\sum_{i<j} \chi_{i} A_{i j} \chi_{j}+\sum_{i<j} \omega_{i} A_{i j} \omega_{j}} .
$$

Proof The proof consists in performing the change of variables 1.63 and canceling carefully the $i$ factors.

There are also normalized Grassmann Gaussian integrals which may be expressed formally as

$$
d \mu_{M}=\frac{\prod d \bar{\psi}_{i} d \psi_{i} e^{-\sum_{i j} \bar{\psi}_{i} M_{i j}^{-1} \psi_{j}}}{\int \prod d \bar{\psi}_{i} d \psi_{i} e^{-\sum_{i j} \bar{\psi}_{i} M_{i j}^{-1} \psi_{j}}} .
$$

and again are characterized by their two point function or covariance

$$
\int \bar{\psi}_{i} \psi_{j} d \mu_{M}=M_{i j}
$$

plus the Grassmann-Wick rule that $n$-point functions are expressed as sum over Wick contractions with signs.

For a much more detailed introduction to the rules of Grassmann calculus in QFT, we refer to [41]. 


\subsection{Trees, forests and the parametric representation}

Classical evolution can be expanded perturbatively into sums indexed by trees whether in quantum field theory the loops of Feynman graphs are essential.

The hidden trees of the classical system inside QFT can be revealed only under scale analysis, since they do not correspond to ordinary spanning trees of the graphs, but to the abstract inclusion relations of short range effects (high energy quasi local subgraphs) inside larger ones. This point of view has been progressively formalized over the years from Bogoliubov to Zimmermann to the most recent formalization by D. Kreimer and A. Connes in terms of Hopf algebras.

But ordinary spanning trees of a connected graph also enter in a fascinating way in the computation of its amplitude. Since the heat kernel is quadratic it is possible to explicitly compute all spatial integrations in a Feynman amplitude. One obtains the so-called parametric representation. The result is expressed in terms of topological or so-called "Symanzik" polynomials [23, 24.

The amplitude of an amputated graph $G$ with external momenta $p$ is, up to a normalization, in space-time dimension $D$ :

$$
A_{G}(p)=\delta\left(\sum p\right) \int_{0}^{\infty} \frac{e^{-V_{G}(p, \alpha) / U_{G}(\alpha)}}{U_{G}(\alpha)^{D / 2}} \prod_{l}\left(e^{-m^{2} \alpha_{l}} d \alpha_{l}\right) .
$$

The first and second Symanzik polynomials $U_{G}$ and $V_{G}$ are

$$
\begin{aligned}
U_{G} & =\sum_{T} \prod_{l \notin T} \alpha_{l}, \\
V_{G} & =\sum_{T_{2}} \prod_{l \notin T_{2}} \alpha_{l}\left(\sum_{i \in E\left(T_{2}\right)} p_{i}\right)^{2},
\end{aligned}
$$

where the first sum is over spanning trees $T$ of $G$ and the second sum is over two trees $T_{2}$, i.e. forests separating the graph in exactly two connected components $E\left(T_{2}\right)$ and $F\left(T_{2}\right)$; the corresponding Euclidean invariant $\left(\sum_{i \in E\left(T_{2}\right)} p_{i}\right)^{2}$ is, by momentum conservation, also equal to $\left(\sum_{i \in F\left(T_{2}\right)} p_{i}\right)^{2}$.

The proof of relations 1.69a $1.69 \mathrm{~b}$ is a special case of the Tree matrix Theorem, which we now explain following 42 .

Theorem 1.3 (Tree Matrix Theorem) Let $A$ be an $n$ by $n$ matrix such that

$$
\sum_{i=1}^{n} A_{i j}=0 \quad \forall j
$$

Obviously $\operatorname{det} A=0$. But let $A^{11}$ be the matrix $A$ with line 1 and column 1 deleted. Then

$$
\operatorname{det} A^{11}=\sum_{T} \prod_{\ell \in T} A_{i_{\ell}, j_{\ell}}
$$

where the sum runs over all directed trees on $\{1, \ldots, n\}$, directed away from root 1. 
This theorem has both a positivity and a democracy aspect: all trees contribute with positive, equal weights to the determinant.

Proof of Theorem 1.3: We use Grassmann variables to write the determinant of a matrix with one line and one raw deleted as a Grassmann integral with two corresponding sources:

$$
\operatorname{det} A^{11}=\int(\mathrm{d} \bar{\psi} \mathrm{d} \psi)\left(\psi_{1} \bar{\psi}_{1}\right) e^{-\bar{\psi} A \psi}
$$

The trick is to use 1.70 to write

$$
\bar{\psi} A \psi=\sum_{i, j=1}^{n}\left(\bar{\psi}_{i}-\bar{\psi}_{j}\right) A_{i j} \psi_{j}
$$

and to obtain

$$
\begin{aligned}
\operatorname{det} A^{11} & =\int \mathrm{d} \bar{\psi} \mathrm{d} \psi\left(\psi_{1} \bar{\psi}_{1}\right) \exp \left(-\sum_{i, j=1}^{n} A_{i j}\left(\bar{\psi}_{i}-\bar{\psi}_{j}\right) \psi_{j}\right) \\
& =\int \mathrm{d} \bar{\psi} \mathrm{d} \psi\left(\psi_{1} \bar{\psi}_{1}\right)\left[\prod_{i, j=1}^{n}\left(1-A_{i j}\left(\bar{\psi}_{i}-\bar{\psi}_{j}\right) \psi_{j}\right)\right]
\end{aligned}
$$

by the Pauli exclusion principle. We now expand to get

$$
\operatorname{det} A^{11}=\sum_{\mathcal{G}}\left(\prod_{\ell=(i, j) \in \mathcal{G}}\left(-A_{i j}\right)\right) \Omega_{\mathcal{G}}
$$

where $\mathcal{G}$ is any subset of $[n] \times[n]$, and we used the notation

$$
\Omega_{\mathcal{G}} \stackrel{\text { def }}{=} \int \mathrm{d} \bar{\psi} \mathrm{d} \psi\left(\psi_{1} \bar{\psi}_{1}\right)\left(\prod_{(i, j) \in \mathcal{G}}\left[\left(\bar{\psi}_{i}-\bar{\psi}_{j}\right) \psi_{j}\right]\right)
$$

The tree matrix theorem then follows from the following

Lemma $1.4 \Omega_{\mathcal{G}}=0$ unless the graph $\mathcal{G}$ is a tree directed away from 1 in which case $\Omega_{\mathcal{G}}=1$.

Proof of the lemma : Trivially, if $(i, i)$ belongs to $\mathcal{G}$, then the integrand of $\Omega_{\mathcal{G}}$ contains a factor $\bar{\psi}_{i}-\bar{\psi}_{i}=0$ and therefore $\Omega_{\mathcal{G}}$ vanishes.

But the crucial observation is that if there is a loop in $\mathcal{G}$ then again $\Omega_{\mathcal{G}}=0$. This is because then the integrand of $\Omega_{\mathcal{F}, \mathcal{R}}$ contains the factor

$$
\bar{\psi}_{\tau(k)}-\bar{\psi}_{\tau(1)}=\left(\bar{\psi}_{\tau(k)}-\bar{\psi}_{\tau(k-1)}\right)+\cdots+\left(\bar{\psi}_{\tau(2)}-\bar{\psi}_{\tau(1)}\right)
$$

Now, upon inserting this telescoping expansion of the factor $\bar{\psi}_{\tau(k)}-\bar{\psi}_{\tau(1)}$ into the integrand of $\Omega_{\mathcal{F}, \mathcal{R}}$, the latter breaks into a sum of $(k-1)$ products. For 
each of these products, there exists an $\alpha \in \mathbb{Z} / k \mathbb{Z}$ such that the factor $\left(\bar{\psi}_{\tau(\alpha)}-\right.$ $\left.\bar{\psi}_{\tau(\alpha-1)}\right)$ appears twice : once with the + sign from the telescopic expansion of $\left(\bar{\psi}_{\tau(k)}-\bar{\psi}_{\tau(1)}\right)$, and once more with a + (resp. -$)$ sign if $(\tau(\alpha), \tau(\alpha-1))$ (resp. $(\tau(\alpha-1), \tau(\alpha)))$ belongs to $\mathcal{F}$. Again, the Pauli exclusion principle entails that $\Omega_{\mathcal{G}}=0$.

Now every connected component of $\mathcal{G}$ must contain 1, otherwise there is no way to saturate the $d \psi_{1}$ integration.

This means that $\mathcal{G}$ has to be a directed tree on $\{1, \ldots n\}$. It remains only to see that $\mathcal{G}$ has to be directed away from 1 , which is not too difficult.

Relations 1.69a $1.69 \mathrm{~b}$ follow rather easily from the tree matrix theorem and the direct representation of Feynman amplitudes (1.80).

In [21] a deeper proof of these relations is given. It relies on the more canonical phase-space parametric representation, which we briefly describe now. Let us limit ourselves to "semi-regular" graphs, which have no "tadpoles" that it no line starting and ending at the same vertex. These graphs (once their lines have been oriented in an arbitrary way) are nicely characterized by their incidence matrix, which is a regular $l(G)$ by $n(G)$ matrix $\epsilon_{\ell v}$ with

$$
\begin{aligned}
& \epsilon_{\ell v}=-1 \text { if line } \ell \text { exits vertex } v \\
& \epsilon_{\ell v}=+1 \text { if line } \ell \text { enters vertex } v \\
& \epsilon_{\ell v}=0 \text { otherwise }
\end{aligned}
$$

There are also external momenta $p_{f}, f=1, \cdots, N$, which we could also also orient through a matrix $\epsilon_{f v}$.

The momentum parametric representation then writes

$A_{G}^{T}\left(p_{1}, \ldots, p_{N}\right)=\delta\left(\sum_{f, v} \epsilon_{f v} p_{f}\right) \int \prod_{\ell=1}^{l(G)} d \alpha_{\ell} d^{d} k_{\ell} e^{-\alpha_{\ell}\left(k_{\ell}^{2}+m^{2}\right)} \prod_{v=1}^{n(G)-1} \delta\left(\epsilon_{f v} p_{f}+\epsilon_{\ell v} k_{\ell}\right)$.

But there is no canonical way to solve for the delta functions, something known in physics as the procedure of momentum attribution. So it is better to rewrite these amplitudes in the phase-space parametric representation

$$
A_{G}^{T}\left(p_{1}, \ldots, p_{N}\right)=\int \prod_{\ell=1}^{l(G)}\left[d \alpha_{\ell} e^{-\alpha_{\ell} m^{2}} d^{d} k_{\ell}\right] \prod_{v=1}^{V-1} d^{d} x_{v} e^{-\alpha_{\ell} k_{\ell}^{2}+2 i\left(p_{f} \epsilon_{v f} x_{v}+k_{\ell} \epsilon_{v \ell} x_{v}\right)},
$$

Integrating over momenta leads to the direct space parametric representation:

$$
A_{G}^{T}\left(p_{1}, \ldots, p_{N}\right)=\int \prod_{\ell=1}^{l(G)} d \alpha_{\ell} \frac{e^{-\alpha_{\ell} m^{2}}}{\alpha_{\ell}^{d / 2}} \prod_{v=1}^{n(G)-1} d^{d} x_{v} e^{2 i p_{f} \epsilon_{v f} x_{v}-x_{v} \cdot x_{v^{\prime}} \epsilon_{\ell v} \epsilon_{\ell v^{\prime}} / \alpha_{\ell}} .
$$

In 21] it is shown how the representation 1.80 together with the quasiPfaffian representation of Lemma 1.2 leads to deletion-contraction relations for the Symanzik polynomials which allow to compute $1.69 \mathrm{a} 1.69 \mathrm{~b}$ from the theory of the universal Tutte polynomial. 


\subsection{BKAR Forest Formula}

Since we want to implement Renormalization Group in a non-perturbative or constructive way, we need tools to compute connected functions in a nonperturbative way, with the right scaling properties for the convergence radius of the expansion. For instance in the just renormalizable case, we need a convergence radius in the coupling constant which is uniform in the scale index.

The main such tool is a canonical forest formula [38, 39] which allows to package a perturbative expansion in terms of trees rather than Feynman graphs. The advantage was already mentioned several times: the species of trees does not proliferate [19, 20] at large orders, in contrast with the species of Feynman graphs.

Consider $n$ points; the set of pairs $P_{n}$ of such points which has $n(n-1) / 2$ elements $\ell=(i, j)$ for $1 \leq i<j \leq n$, and a smooth function $f$ of $n(n-1) / 2$ variables $x_{\ell}, \ell \in \mathcal{P}_{n}$. Noting $\partial_{\ell}$ for $\frac{\partial}{\partial x_{\ell}}$, the standard forest formula is

$$
f(1, \ldots, 1)=\sum_{\mathcal{F}}\left[\prod_{\ell \in \mathcal{F}} \int_{0}^{1} d w_{\ell}\right]\left(\left[\prod_{\ell \in \mathcal{F}} \partial_{\ell}\right] f\right)\left[x_{\ell}^{\mathcal{F}}\left(\left\{w_{\ell^{\prime}}\right\}\right)\right]
$$

where

- the sum over $\mathcal{F}$ is over forests over the $n$ vertices, including the empty one

- $x_{\ell}^{\mathcal{F}}\left(\left\{w_{\ell^{\prime}}\right\}\right)$ is the infimum of the $w_{\ell^{\prime}}$ for $\ell^{\prime}$ in the unique path from $i$ to $j$ in $\mathcal{F}$, where $\ell=(i, j)$. If there is no such path, $x_{\ell}^{\mathcal{F}}\left(\left\{w_{\ell^{\prime}}\right\}\right)=0$ by definition.

- The symmetric $n$ by $n$ matrix $X^{\mathcal{F}}(\{w\})$ defined by $X_{i i}^{\mathcal{F}}=1$ and $X_{i j}^{\mathcal{F}}=$ $x_{i j}^{\mathcal{F}}\left(\left\{w_{\ell^{\prime}}\right\}\right)$ for $1 \leq i<j \leq n$ is positive.

This formula can be viewed as a tool to associate to any pair made of a graph $G$ and a spanning forest $F \subset G$ a unique rational number or weight $w(G, F)$ between 0 and 1, called the relative weight of $T$ in $G$. These weights are barycentric or percentage factors, ie for any $G$

$$
\sum_{F \subset G} w(G, F)=1
$$

The numbers $w(G, F)$ are multiplicative over disjoint unions ${ }^{5}$. Hence it is enough to give the formula for $(G, F)$ only when $G$ is connected and $F=T$ is a spanning tree in it ${ }^{6}$. The definition of these weights is

\section{Definition 1}

$$
w(G, T)=\prod_{\ell \in T} \int_{0}^{1} \prod_{\ell \in T} d w_{\ell} \prod_{\ell \notin T} x_{\ell}^{T}(\{w\})
$$

where $x_{\ell}^{T}(\{w\})$ is again the infimum over the $w_{\ell^{\prime}}$ parameters over the lines $\ell^{\prime}$ forming the unique path in $T$ joining the ends of $\ell$.

\footnotetext{
${ }^{5}$ And also over vertex joints of graphs, just as in the universality theorem for the Tutte polynomial

${ }^{6}$ It is enough in fact to compute such weights for 1-particle irreducible and 1-vertexirreducible graphs, then multiply them in the appropriate way for the general case.
} 
Consider the expansion in terms of Feynman amplitudes of a connected quantity $S$. The most naive way to reorder Feynman perturbation theory according to trees rather than graphs is to insert for each graph the relation (1.81)

$$
S=\sum_{G} A_{G}=\sum_{G} \sum_{T \subset G} w(G, T) \mathcal{A}_{G}
$$

and exchange the order of the sums over $S$ and $T$. Hence it writes

$$
S=\sum_{T} \mathcal{A}_{T}, \quad \mathcal{A}_{T}=\sum_{G \supset T} w(G, T) \mathcal{A}_{G} .
$$

This rearranges the Feynman expansion according to trees, but each tree has the same number of vertices as the initial graph. Hence it reshuffles the various terms of a given, fixed order of perturbation theory. Remark that if the initial graphs have say degree 4 at each vertex, only trees with degree less than or equal to 4 occur in the rearranged tree expansion.

For Fermionic theories this is typically sufficient and one has for small enough coupling

$$
\sum_{T}\left|\mathcal{A}_{T}\right|<\infty
$$

because Fermionic graphs essentially mostly compensate each other at a fixed order by Pauli's principle; mathematically this is because these graphs form a determinant and the size of a determinant is much less than what its permutation expansion usually suggests. This is well known [46, 62, 47.

But this repacking fails completely for Bosonic theories, because the only compensations there occur between graphs of different orders. Hence if we perform this naive reshuffling, eg on the $\phi_{0}^{4}$ theory we would still have

$$
\sum_{T}\left|\mathcal{A}_{T}\right|=\infty .
$$

Recently a new expansion called the Loop Vertex Expansion has been found [16] which overcomes this difficulty by exchanging the role of vertices and propagators before applying the forest formula. It can also be seen as a combination of the forest formula with the so-called intermediate field representation, which expands into essentially square roots of a stable Bosonic interaction. We refer the reader to [16, 17, 19, 40, 20 but wont review this expansion here, since from now on we are mostly going to deal with Fermions.

\subsection{Gram and Hadamard Bounds}

These two bounds on a determinant are often confused!

The Gram bound applies to a matrix $A=a_{i j}$ whose entries are scalar product. This means we suppose that there exists some Hilbert space $H$ and $2 n$ vectors $f_{i}, i=1, \cdots, n, g_{j}, j=1, \cdots, n$ with

$$
a_{i j}=<f_{i}, g_{j}>_{H} .
$$


The Gram bound states

$$
|\operatorname{det} A| \leq \prod_{i=1}^{n}\left\|f_{i}\right\|_{H} \prod_{j=1}^{n}\left\|g_{j}\right\|_{H} .
$$

Of course any matrix $A$ can always be written of the Gram type, eg with $H=\mathbb{R}^{n}, f_{i}=\left(a_{i, k}\right)$ and $g_{j}=\delta_{j, k}, k=1, \cdots, n$, or conversely. Hence there are two corresponding asymmetric Hadamard bounds, one for rows and one for columns:

$$
\begin{aligned}
& |\operatorname{det} A| \leq \prod_{i=1}^{n} \sqrt{\sum_{j=1}^{n} a_{i j}^{2}}, \\
& |\operatorname{det} A| \leq \prod_{j=1}^{n} \sqrt{\sum_{i=1}^{n} a_{i j}^{2}}
\end{aligned}
$$

and also a symmetric Hadamard bound involving the supremum of the matrix elements:

$$
|\operatorname{det} A| \leq n^{n / 2}\left(\sup _{i, j}\left|a_{i j}\right|\right)^{n} .
$$

Remark that the symmetric Hadamard bound means that a determinant of a large matrix is always much smaller than what its permutation expansion plus naive bounds would suggest, which is the "stupid bound"

$$
|\operatorname{det} A| \leq n !\left(\sup _{i, j}\left|a_{i j}\right|\right)^{n} .
$$

This difference in constructive theory is essential. Indeed for Fermionic theories with bounded propagators and a quartic interaction, the matrix $A$ at $n$-th order of perturbation is a $2 n \times 2 n$ matrix, with propagators as matrix elements, and there is a $1 / n$ ! symmetry factor. Hence the bound 1.92 would lead to believe that the radius of convergence of the partition function is 0 , like in the Bosonic case. But the Hadamard bound (1.91) proves that it is at least finite. Moreover usually it is possible to write the propagators as scalar products in $L^{2}(\mathbb{R})^{d}$ of functions which also have bounded $L^{2}$ norm 7 . In that case the Gram bound (1.88) shows that the partition function is in fact an entire function, as it shows no factorial dependence at all as $n \rightarrow \infty$ !

\subsection{Single Scale Constructive Theory for a Toy Model}

Consider a just-renormalizable QFT theory. The key problem is to compute connected quantities with an expansion which converges for a small coupling constant, with a propagator limited to a single renormalization group scale, uniformly in the slice index. In the simple Fermionic case, this can be done through applying first the BKAR formula then checking convergence through a Gram bound.

\footnotetext{
${ }^{7}$ This is usually easily done by taking some kind of "square roots" in momentum space.
} 
To discuss the type of Fermionic models met in condensed matter it is appropriate to consider first a toy Fermionic $d$-dimensional QFT model. It is made of a single an infrared slice with $N$ colors. Suppose the propagator is diagonal in color space and satisfies the bound

$$
\left|C_{j, a b}(x, y)\right| \leq \delta_{a b} \frac{M^{-d j / 2}}{\sqrt{N}} e^{-M^{-j}|x-y|} .
$$

We say that the interaction is of the vector type (or Gross-Neveu type) if it is of the form

$$
V=\lambda \int d^{d} x\left(\sum_{a=1}^{N} \bar{\psi}_{a}(x) \psi_{a}(x)\right)\left(\sum_{b=1}^{N} \bar{\psi}_{b}(x) \psi_{b}(x)\right)
$$

where $\lambda$ is the coupling constant.

We claim that

Lemma 1.5 The perturbation theory for the connected functions of this single slice model has a radius of convergence in $\lambda$ which is uniform in $j$ and $N$.

To prove this lemma, expands the partition function $Z(\Lambda)$ through the forest formula, and take the logarithm to obtain a tree formula for the pressure

$$
p=\lim _{\Lambda \rightarrow \infty} \frac{1}{|\Lambda|} \log Z(\Lambda) .
$$

This is completely straightforward, the only difficulty being notational. Using the notations of 47

$$
\begin{aligned}
& p=\lim _{\Lambda \rightarrow \infty} \frac{1}{|\Lambda|}\left(\int d \mu_{C}(\psi, \bar{\psi}) e^{S_{\Lambda}\left(\bar{\psi}_{a}, \psi_{a}\right)}\right) \\
& =\sum_{n=0}^{\infty}\left(\lambda^{n} / N^{n} n !\right) \sum_{a_{1}, \ldots, a_{n}, b_{1}, \ldots, b_{n}=1}^{N} \sum_{\mathcal{T}} \sum_{\Omega} \epsilon(\mathcal{T}, \Omega)\left(\prod_{l \in \mathcal{T}} \int_{0}^{1} d w_{l}\right) \\
& \int_{\mathbb{R}^{n d}} d x_{1} \ldots d x_{n} \delta\left(x_{1}=0\right) \prod_{l \in \mathcal{T}}\left(C\left(x_{i(l)}, x_{j(l)}\right) \delta_{l}\right) \times \operatorname{det}_{\text {remaining }}\left(C_{\alpha \beta}\right)_{\alpha \in A, \beta \in B}
\end{aligned}
$$

The sum over the $a_{i}$ 's and $b_{i}$ 's are over the colors of the fields and antifields of the vertices obtained by expanding the interaction and of the form:

$$
\bar{\psi}_{a_{i}}\left(x_{i}\right) \psi_{a_{i}}\left(x_{i}\right) \bar{\psi}_{b_{i}}\left(x_{i}\right) \psi_{b_{i}}\left(x_{i}\right)
$$

with $1 \leq i \leq n$. The sum over $\mathcal{T}$ is over all trees which connect together the $n$ vertices at $x_{1}, \ldots, x_{n}$. The sum over $\Omega$ is over the compatible ways of realizing the bonds $l=\{i, j\} \in \mathcal{T}$ as contractions of a $\psi$ and $\bar{\psi}$ between the vertices $i$ and $j$ (compatible means that we do not contract twice the same field or antifield). $\epsilon(\mathcal{T}, \Omega)$ is a sign which is not important for the bound (see [47] for its explicit computation). For any $l \in \mathcal{T}, i(l) \in\{1, \ldots, n\}$ labels the vertex where the field, 
contracted by the procedure $\Omega$ concerning the link $l$, was chosen. Likewise $j(l)$ is the label for the vertex containing the contracted antifield. $\delta_{l}$ is 1 if the colors (among $a_{1}, \ldots, a_{n}, b_{1}, \ldots, b_{n}$ ) of the field and antifield contracted by $l$ are the same and else is 0 . Finally the matrix $\left(C_{\alpha \beta}\right)_{\alpha, \beta}$ of the remaining "loop lines" is defined in the following manner.

The row indices $\alpha$ label the $2 n$ fields produced by the $n$ vertices, so that $\alpha=(i, \sigma)$ with $1 \leq i \leq n$ and $\sigma$ takes two values 1 or 2 to indicate whether the field is the second or the fourth factor in (1.97) respectively.

The column indices $\beta$ label in the same way the $2 n$ antifields, so that $\beta=$ $(j, \tau)$ with $1 \leq j \leq n$ and $\tau=1$ or 2 according to whether the antifield is the first or the third factor in $(1.97)$ respectively. The $\alpha$ 's and $\beta$ 's are ordered lexicographically. We denote by $c(i, \sigma)$ the color of the field labeled by $(i, \sigma)$ that is $a_{i}$, if $\sigma=1$, and $b_{i}$ if $\sigma=2$. We introduce the similar notation $\bar{c}(j, \tau)$ for the color of an antifield. Now

$$
C_{(i, \sigma)(j, \tau)}=w_{i j}^{\mathcal{T}, B K}(\mathbf{w}) C\left(x_{i}, x_{j}\right) \delta(c(i, \sigma), \bar{c}(j, \tau)) .
$$

Finally each time a field $(i, \sigma)$ is contracted by $\Omega$ the corresponding row is deleted from the $2 n \times 2 n$ matrix $\left(C_{\alpha \beta}\right)$. Likewise, for any contracted antifield the corresponding column is erased. $A$ and $B$ denote respectively the set of remaining rows and the set of remaining columns. The minor determinant featuring as det $\operatorname{demaining}_{\text {rem }}$ in is now $\operatorname{det}\left(b_{\alpha \beta}\right)_{\alpha \in A, \beta \in B}$ which is $(n+1) \times$ $(n+1)$. Indeed for each of the $n-1$ links of $\mathcal{T}$, a row and a column have been erased.

Suppose we have written

$$
C_{j}\left(x_{k}, y_{m}\right)<f_{j, k}, g_{j, m}>_{L^{2}}
$$

(this is realized through $f_{j, k}=f_{j}\left(x_{k}, \cdot\right)$ and $g_{j, m}=g_{j}\left(\cdot, y_{m}\right)$ if $\hat{f}_{j}(p) . \hat{g}_{j}(p)=$ $\left.\hat{C}_{j}(p)\right)$. and that the power counting is conserved by taking square-roots, namely that the $L^{2}$ norms of $g_{j}$ and $f_{j}$ scale as $\frac{M^{-d j / 4}}{N^{1 / 4}}$.

Then applying the Gram inequality

$$
\left|\operatorname{det}\left[C_{j, a b}()\right]_{\text {remaining }}\right| \leq \prod_{\text {anti-fields } k}\left\|f_{j, k}\right\| \prod_{\text {fields } m}\left\|g_{j, m}\right\|
$$

will lead to the proof that the radius of convergence of the pressure is uniform in $j$ and $N$ for this toy model.

Indeed the following lemma shows that the presence of the weakening factors $w$ does not change the outcome of the Gram bound.

Lemma Let $\mathcal{A}=\left(a_{\alpha \beta}\right)_{\alpha, \beta}$ be a Gram matrix: $a_{\alpha \beta}=<f_{\alpha}, g_{\beta}>$ for some inner product $\langle., .>$. Suppose each of the indices $\alpha$ and $\beta$ is of the form $(i, \sigma)$ where the first index $i, 1 \leq i \leq n$, has the same range as the indices of the positive matrix $\left(w_{i j}^{\mathcal{F}, B K}(\mathbf{w})\right)_{i j}$, and $\sigma$ runs through some other index set $\Sigma$.

Let $\mathcal{C}=\left(C_{\alpha \beta}\right)_{\alpha, \beta}$ be the matrix with entries $C_{(i, \sigma)(j, \tau)}=w_{i j}^{\mathcal{F}, B K}(\mathbf{w}) .<$ $f_{(i, \sigma)}, g_{(j, \tau)}>$ and let $\left(C_{\alpha \beta}\right)_{\alpha \in A, \beta \in B}$ be some square matrix extracted from $\mathcal{C}$, 
then for any $\mathbf{w}$ we have the Gram inequality:

$$
\left|\operatorname{det}\left(C_{\alpha \beta}\right)_{\alpha \in A, \beta \in B}\right| \leq \prod_{\alpha \in A}\left\|f_{\alpha}\right\| \prod_{\beta \in B}\left\|g_{\beta}\right\|
$$

Proof Indeed we can take the symmetric square root $v$ of the positive matrix $w^{\mathcal{F}, B K}$ so that $w_{i j}^{\mathcal{F}, B K}=\sum_{k=1}^{n} v_{i k} v_{k j}$. Let us denote the components of the vectors $f$ and $g$, in an orthonormal basis for the scalar product $\langle.,$.$\rangle with$ $q$ elements, by $f_{(i, \sigma)}^{m}$ and $g_{(j, \tau)}^{m}, 1 \leq m \leq q$. (Indeed even if the initial Hilbert space is infinite dimensional, the problem is obviously restricted to the finite dimensional subspace generated by the finite set of vectors $f$ and $g$ ). We then define the tensorized vectors $F_{(i, \sigma)}$ and $G_{(j, \tau)}$ with components $F_{(i, \sigma)}^{k m}=v_{i k} f_{(i, \sigma)}^{m}$ and $G_{(j, \tau)}^{k m}=v_{j k} g_{(j, \tau)}^{m}$ where $1 \leq k \leq n$ and $1 \leq m \leq q$. Now considering the tensor scalar product $<., .>_{T}$ we have

$$
<F_{(i, \sigma)}, G_{(j, \tau)}>_{T}=\sum_{k=1}^{n} \sum_{m=1}^{q} v_{i k} v_{j k} f_{(i, \sigma)}^{m} g_{(j, \tau)}^{m}=b_{(i, \sigma)(j, \tau)} .
$$

By Gram's inequality using the $<., .>_{T}$ scalar product we get

$$
\left.\mid \operatorname{det}\left(C_{\alpha \beta}\right)_{\alpha \in A, \beta \in B}\right) \mid \leq \prod_{\alpha \in A}\left\|f_{\alpha}\right\|_{T} \prod_{\beta \in B}\left\|g_{\beta}\right\|_{T}
$$

but

$$
\begin{aligned}
\left\|F_{(i, \sigma)}\right\|_{T}^{2} & =\sum_{k=1}^{n} \sum_{m=1}^{q}\left(F_{(i, \sigma)}^{k m}\right)^{2}=\sum_{k=1}^{n} \sum_{m=1}^{q} v_{i k}^{2}\left(f_{(i, \sigma)}^{m}\right)^{2} \\
& =w_{i i} \sum_{m=1}^{q}\left(f_{(i, \sigma)}^{m}\right)^{2}=\left\|f_{(i, \sigma)}\right\|^{2}
\end{aligned}
$$

since $w_{i i}=1$ for any $i, 1 \leq i \leq n$.

Let us apply the Gram bound and this last Lemma to bound the determinant in (1.97).

- There is a factor $M^{-d j / 2}$ per line, or $M^{-d j / 4}$ per field ie entry of the loop determinant. This gives a factor $M^{-d j}$ per vertex.

- There is a factor $M^{+d j}$ per vertex spatial integration (minus one)

Hence the $\lambda$ radius of convergence is uniform in $j$.

- There is a factor $N^{-1 / 2}$ per line, or $N^{-1 / 4}$ per field ie entry of the loop determinant. This gives a factor $N^{-1}$ per vertex

- There is a factor $N$ per vertex (plus an extra one) 
This leads to a bound in $N \cdot M^{2 j}[c \lambda]^{n}$ for the $n$-th order of the pressure, hence to a radius of convergence at least $1 / c$. As expected, this is a bound uniform in the slice index $j$. Hence the $\lambda$ radius of convergence is uniform in $N$.

The last item, namely the factor $N^{n+1}$ for the colors sums is the only one not obvious to prove. Indeed don't know all the graph, but only a spanning tree. We need to organize the sum over the colors from the leaves to the root of the tree. In this way the pay a factor $N$ at each leaf to know the color index which does not go towards the root, then prune the leaf and iterate. The last vertex (the root) is the only special one as it costs two $N$ factors.

Let us remark that to treat the corresponding toy model in the Bosonic case the standard constructive method would be to perform a cluster expansion with respect to a lattice of cubes, then a Mayer expansion which further removed the remaining hardcore constraints with respect to the cubes 9 . Both expansions needed to use the forest formula. This was simplified by the invention of the Loop vertex expansion, in which cubes, cluster and Mayer expansions are no longer needed. In addition the Loop vertex model leads to uniform bounds also for matrix toy models, a result which cannot be obtained up to now with other methods 16.

\section{Interacting Fermions in Two Dimensions}

\subsection{Introduction}

One of the main achievements in renormalization theory has been the extension of the renormalization group of Wilson (which analyzes long-range behavior governed by simple scaling around the point singularity $p=0$ in momentum space) to long-range behavior governed by extended singularities [51, 52, 53. This very natural and general idea is susceptible of many applications in various domains, including condensed matter (reviewed here, in which the extended singularity is the Fermi surface) but also other ones such as diffusion in Minkowski space (in which the extended singularity is the mass shell). In this section we will discuss interacting Fermions models such as those describing the conduction electrons in a metal.

The key features which differentiate electrons in condensed matter from Euclidean field theory, and make the subject in a way mathematically richer, is that space-time rotation invariance is broken, and that particle density is finite. This finite density of particles creates the Fermi sea: particles fill states up to an energy level called the Fermi surface.

The field theory formalism is the best tool to isolate fundamental issues such as the existence of non-perturbative effects. In this formalism the usual Hamiltonian point of view with operators creating electrons or holes is superseded by the more synthetic point of view of anticommuting Fermionic fields with two spin indices and arguments in $d+1$ dimensional space-time,. Beware however of the QFT-convention to always call dimension the dimension of space-time, whether from now on we have to stick to the usual condensed matter conven- 
tion which is to always call dimension the dimension of space only. So one dimensional interacting Fermions correspond at zero temperature to a two dimensional QFT, two dimensional Fermions correspond at zero temperature to a three dimensional QFT and so on.

After the discovery of high temperature superconductivity, a key question emerged. Do interacting Fermions in 2 dimensions (above their low-temperature phase) resemble more three dimensional Fermions, i.e. the Fermi liquid, or one dimensional Fermions, i.e. the Luttinger liquid? The short answer to this controversial question is that it was solved rigorously by mathematical physics and that the answer depends on the shape of the Fermi surface. Interacting Fermions with a round Fermi surface behave more like three dimensional Fermi liquids, whether interacting Fermions with the square Fermi surface of the Hubbard model at half-filling behave more like a one-dimensional Luttinger liquid.

This statement has been now proved in full mathematical rigor, beyond perturbation theory, in the series of works [54, 55, 56, 57, 58, 59, 60].

The existence of usual 2D interacting Fermi liquids was established in [54, 55] using the mathematically precise criterion of Salmhofer [48] in the case of a temperature infra-red regulator. Using a magnetic field regulator that breaks parity invariance of the Fermi surface it was also established in the initial sense of a discontinuity at the Fermi surface in the series of papers [4].

\subsection{The Models: $J_{2}, J_{3}, H_{2} \ldots$}

We consider a gas of Fermions in thermal equilibrium at temperature $T$, with coupling constant $\lambda$. The free propagator for this model is

$$
\hat{C}_{a b}(k)=\delta_{a b} \frac{1}{i k_{0}-e(\mathbf{k})}
$$

with $\mathbf{k}$ being the $d$-dimensional momentum, $e(\mathbf{k})=\epsilon(\mathbf{k})-\mu, \epsilon(\mathbf{k})$ being the kinetic energy and $\mu$ the chemical potential. The $a, b \in\{\uparrow, \downarrow\}$ index is for spin hence can take two values (remember spin is treated non-relativistically).

At finite temperature, since Fermionic fields have to satisfy antiperiodic boundary conditions, the component $k_{0}$ in (2.1) can take only discrete values (called the Matsubara frequencies) : so the integral over $k_{0}$ is really a discrete sum over $n$.

These Matsubara frequencies are:

$$
k_{0}=\frac{2 n+1}{\beta \hbar} \pi, \quad n \in \mathbb{Z}
$$

where $\beta=(k T)^{-1}$. For any $n$ we have $k_{0} \neq 0$, so that the denominator in $C(k)$ can never be 0 . This is why the temperature provides a natural infrared cut-off.

We can think of $k_{0}$ as the Fourier dual to an imaginary Euclidean-time continuous variable taking values in a circle, with length proportional to inverse temperature $\beta$. When $T \rightarrow 0^{+}$, (which means $\left.\beta \rightarrow+\infty\right), k_{0}$ becomes a continuous variable, the corresponding discrete sum becomes an integral, and the 
corresponding propagator $C_{0}(x)$ becomes singular on the Fermi surface defined by $k_{0}=0$ and $e(\mathbf{k})=0$.

This Fermi surface depends on the kinetic energy $\epsilon(\mathbf{k})$ of the model.

For rotation invariant models, $\epsilon(\mathbf{k})=\mathbf{k}^{2} / 2 m$ where $m$ is some effective or "dressed" electron mass. In this case the energy is invariant under spatial rotations and the Fermi surface is simply a circle in two dimensions and a sphere in three dimensions, with radius $\sqrt{2 m \mu}$. This jellium isotropic propagator is realistic in the limit of weak electron densities. We call this propagator the jellium propagator. We always consider this model, the most natural one in the continuum, together with an ultraviolet cutoff (which it is natural to also take rotation invariant $)^{8}$

Another model considered extensively is the half-filled $2 d$ Hubbard model, nicknamed $H_{2}$. In this model the position variable $\mathbf{x}$ lives on the lattice $\mathbb{Z}^{2}$, and $\epsilon(\mathbf{k})=\cos k_{1}+\cos k_{2}$ so that at $\mu=0$ the Fermi surface is a square of side size $\sqrt{2 \pi}$, joining the points $(\pi, 0),(0, \pi)$ in the first Brillouin zone.

This propagator is called the Hubbard propagator.

\subsection{Interaction, Locality}

The physical interaction between conduction electrons in a solid could be very complicated; the naive Coulomb interaction is in fact subject to heavy screening, the main effective interaction being due to lattice phonons exchange and other effects. But we are interested in long-range physics, Hence we should use a quasi-local action which decays It is a bit counterintuitive but in fact perfectly reasonable for a mathematical idealization to use in fact a fully local interaction. This should capture all essential mathematical difficulties of the corresponding renormalization group.

But there is a unique exactly local such interaction, namely

$$
S_{V}=\lambda \int_{V} d^{d+1}\left(\sum_{a \in\{\uparrow, \downarrow\}} \bar{\psi}_{a}(x) \psi_{a}(x)\right)^{2},
$$

where $V:=\left[-\beta, \beta\left[\times V^{\prime}\right.\right.$ and $V^{\prime}$ is an auxiliary volume cutoff in two dimensional space, that will be sent to infinity in the thermodynamic limit. Indeed any local polynomial of higher degree is zero since Fermionic fields anticommute. Remark it is of the same form than (??), with spin playing the role of color.

Hence from the mathematical point of view, in contrast with the propagator, the interesting condensed matter interaction is essentially unique.

The models with jellium propagator and such an interaction $(2.3)$ are respectively nicknamed $J_{2}$ and $J_{3}$ in dimensions 2 and 3 . The model with Hubbard propagator and interaction 2.3 is nicknamed $\mathrm{H}_{2}$.

It is possible to interpolate continuously between $\mathrm{H}_{2}$ and $J_{2}$ by varying the filling factor of the Hubbard model. Lattice models with next-nearest neighbor

\footnotetext{
8 The question of whether and how to remove that ultraviolet cutoff has been discussed extensively in the literature, but we consider it as unphysical for a non-relativistic model of condensed matter, which is certainly an effective theory at best.
} 
hopping are also interesting, as they are really the ones used to model the high $T_{c}$ superconducting phase in cuprates, but we shall not consider them here for simplicity.

The basic new feature which changes dramatically the power counting of the theory is that the singularity of the jellium propagator is of codimension 2 in the $d+1$ dimensional space-time. Instead of changing with dimension, like in ordinary field theory, perturbative power counting is now independent of the dimension, and is the one of a just renormalizable theory. Indeed in a graph with 4 external legs, there are $n$ vertices, $2 n-2$ internal lines and $L=n-1$ independent loops. Each independent loop momentum gives rise to two transverse variables, for instance $k_{0}$ and $|\mathbf{k}|$ in the jellium case, and to $d-1$ inessential bounded angular variables. Hence the $2 L=2(n-1)$ dimensions of integration for the loop momenta exactly balance the $2 n-2$ singularities of the internal propagators, as is the case in a just renormalizable theory.

In one spatial dimension, hence two space-time dimensions, the Fermi surface reduces to two points, and there is also no proper BCS theory since there is no continuous symmetry breaking in two dimensions (by the "Mermin-Wagner theorem"). Nevertheless the many Fermion system in one spatial dimension gives rise to an interesting non-trivial behavior, called the Luttinger liquid [43].

The marvel is that although the renormalization group now pinches a non trivial extended singularity, the locality principle still works. The leading part of the four point quasi-local graphs is still local, like the interaction of the initial theory. This is very surprising since quasi-local graphs have internal lines which simply carry excitations farther from the Fermi surface than their external ones. Momenta close to the Fermi surface, when moved to a barycenter of the graph, should react through a non trivial phase factor. But the miracle is that the only divergent part of the main one-loop contribution, the "bubble graph" comes when the combination of the two external legs at each end carries approximately zero total momentum. This is because only then can the two inner bubble propagators both range over the full Fermi sphere. This special configuration, with proper spin and arrows to ensure no oscillations occur, is really the Cooper pair!

Then these two external legs with approximately zero total momentum can be moved together like a single low momentum leg in an ordinary Wilsonian renormalization. This is in essence why renormalization still works in condensed matter and beyond the two point function renormalization (Fermi radius renormalization) only changes the value of the coupling constant for the local interaction (2.3). Of course if contributions beyond one loop are taken into account, the story becomes more complicated and the renormalization group flow can in fact involve infinitely many coupling constants [64].

A still more surprising case where locality works in a new form, called Moyality, is the case of non commutative field theory on Moyal space 34. There again the divergent subgraphs are exactly the only ones that can be renormalized through counterterms of the initial form of the Lagrangian [50]. This lead us to hope that another still generalized form of locality might hold in quantum gravity. 


\subsection{A Brief Review of Rigorous Results}

What did the programs of rigorous mathematical study of interacting Fermi systems accomplish until now? Recall that in dimension 1 there is neither superconductivity nor extended Fermi surface, and Fermion systems have been proved to exhibit Luttinger liquid behavior [43. The initial goal of the studies in two or three dimensions was to understand the low temperature phase of these systems, and in particular to build a rigorous constructive BCS theory of superconductivity. The mechanism for the formation of Cooper pairs and the main technical tool to use (namely the corresponding $1 / \mathrm{N}$ expansion, where $\mathrm{N}$ is the number of sectors which proliferate near the Fermi surface at low temperatures) have been identified 62. But the goal of building a completely rigorous BCS theory ab initio remains elusive because of the technicalities involved with the constructive control of continuous symmetry breaking. So the initial goal was replaced by a more modest one, still important in view of the controversies over the nature of two dimensional Fermi liquids 61, namely the rigorous control of what occurs before pair formation.

As is well known, sufficiently high magnetic field or temperature are the two different ways to break the Cooper pairs and prevent superconductivity. Accordingly two approaches were devised for the construction of "Fermi liquids. One is based on the use of non-parity invariant Fermi surfaces to prevent pair formation. These surfaces occur physically when generic magnetic fields are applied to two dimensional Fermi systems. In the large series of papers [49], the construction of two dimensional Fermi liquids for a wide class of non-parity invariant Fermi surfaces has been completed in great detail by Feldman, Knörrer and Trubowitz. These papers establish Fermi liquid behavior in the traditional sense of physics textbooks, namely as a jump of the density of states at the Fermi surface at zero temperature, but they do not apply to the simplest Fermi surfaces, such as circles or squares, which are parity invariant.

The other approach is based on Salmhofer's criterion, in which temperature is the cutoff which prevents pair formation. The corresponding program studies whether given models satisfy Salmhofer's criterion or not. The study of each model has been divided into two main steps of roughly equal difficulty, the control of convergent contributions and the renormalization of the two point functions. In dimension two the corresponding analysis has been completed for $J_{2}$, a Fermi liquid in the sense of Salmhofer, and for $\mathrm{H}_{2}$ which is not, and is a Luttinger liquid with logarithmic corrections, according to [54, 55, 56, 57, 58.

Similar results similar have been also obtained for more general convex curves not necessarily rotation invariant such as those of the Hubbard model at low filling, where the Fermi surface becomes more and more circular, including an improved treatment of the four point functions leading to better constants [59, 60. Therefore as the filling factor of the Hubbard model is moved from halffilling to low filling, we conclude that there must be a crossover from Luttinger liquid behavior to Fermi liquid behavior. This sheds light on the controversy [61] over the Luttinger or Fermi nature of two-dimensional many-Fermion systems above their critical temperature. 


\subsection{Multiscale Analysis, Angular Sectors}

For any two-dimensional model built until now in the constructive sense, the strategy is the same. It is based on some kind of multiscale expansion, which keeps a large fraction of the theory in unexpanded determinants. The global bound on these determinant (using determinant inequalities such as Gram inequality) is much better than if the determinant was expanded into Feynman graphs which would then be bounded one by one, and the bounds summed. The bound obtained in this way would simply diverge at large order (i.e. not prove any analyticity at all in the coupling constant) simply because there are too many Feynman graphs at large order. But the divergence of a bound does not mean the divergence of the true quantity if the bound is bad. Constructive analysis, which keeps loops unexpanded is the correct way to obtain better bounds, which do prove that the true series in fact does not diverge, i.e. has a finite convergence radius in the coupling constant. This radius however shrink when the temperature goes to 0 , and a good constructive analysis should establish the correct shrinking rate, which is logarithmic. This is where multiscale rather than single scale constructive analysis becomes necessary.

The basic idea of the multiscale analysis is to slice the propagator according to the size of its denominator so that the slice with index $j$ corresponds to $\left|i k_{0}+e(\mathbf{k})\right| \simeq M^{-j}$, where $M$ is some fixed constant.

This multiscale analysis is supplemented within each scale by an angular "sector analysis. The number of sectors should be kept as small as possible, so each sector should be as large as possible in the directions tangent to the Fermi surface in three dimensions, or to the Fermi curve in two dimensions. What limits however the size of these sectors is the curvature of the surface, so that stationary phase method could still relate the spatial decay of a propagator within a sector to its dual size in momentum space. In the case of a circle, the number of sectors at distance $M^{-j}$ of the singularity grows therefore at least

like $M^{j / 2}$, hence like a power of $T$. However for the half-filled Hubbard model, since the curvature is "concentrated at the corners the number of sectors grows only like $|\log T|$. In one dimension there are really only two sectors since the Fermi singularity is made of two points. A logarithm is closer to a constant than to a power; this observation is the main reason for which the half-filled Hubbard model is closer to the one-dimensional Luttinger liquid than to the three dimensional Fermi liquid.

Momentum conservation rules for sectors which meet at a given vertex in general are needed to fix the correct power counting of the subgraphs of the model. In the Hubbard case at half filling, these rules are needed only to fix the correct logarithmic power counting, since the growth of sectors near the singularity is only logarithmic. In both cases the net effect in two dimensions of these conservation rules is to roughly identify two pairs of conserved sectors at any vertex, so that in each slice the model resembles an $N$ - component vector model, where $N$ is the number of sectors in the slice.

The multiscale renormalization group analysis of the model then consists essentially in selecting, for any graph, a tree which is a subtree in each of the 
quasi-local connected components of the graph accord- ing to the momentum slicing. These connected components are those for which all internal lines are farther from the Fermi surface than all external lines. The selection of this tree can be performed in a constructive manner, keeping the remaining loop fields in a determinant. The combinatoric difficulty related to the fact that a graph contains many trees is tackled by the forest formula.

Once the scale analysis has been performed, a partial expansion of the loop determinant can detect all the dangerous two and four point functions which require renormalization. A key point is that this expansion can be done without destroying the Gram bound, and the corresponding sum is not too big (this means its cardinal remains bounded by $K^{n}$ (where $K$ is a constant)) because in typical graphs there are not many two and four point subgraphs.

\subsection{One and Two Particle Irreducible Expansions}

Salmhofer's criterion is stated for the self-energy, i.e. the sum of all one-particle irreducible graphs for the two point function. Its study requires the correct renormalization of these contributions. Since angular sectors in a graph may vary from one propagator to the next in a graph, and since different sectors have different decays in different directions, we are in a delicate situation. In order to prove that renormalization indeed does the good that it is supposed to do, one cannot simply rely on the connectedness of these self- energy graphs, but one must use their particle irreducibility explicitly. So the proof requires a constructive particle irreducible analysis of the self-energy. The following the-orem summarizes the results of [54, [55]:

Theorem 2.1 The radius of convergence of the jellium two-dimensional model perturbative series for any thermodynamic function is at least $c /|\log T|$, where $T$ is the temperature and $c$ some numerical constant. As $T$ and $\lambda$ jointly tend to 0 in this domain, the self-energy and its first two momentum derivatives remain uniformly bounded so that the model is a Fermi liquid in the sense of Salmhofer.

In the case of the jellium model $J_{2}$, this analysis can be performed at the level of one-particle irreducible graphs [55. The half-filled Hubbard model, however, is more difficult. Although there is no real divergence of the self-energy (the associated counterterm is zero thanks to the particle hole symmetry of the model at half-filling) one really needs a two-particle and one-vertex irreducible constructive analysis to establish the necessary constructive bounds on the selfenergy and its derivatives [57. For parity reasons, the self-energy graphs of the model are in fact not only one-particle irreducible but also two particle and one vertex irreducible, so that this analysis is possible.

This analysis leads to the explicit construction of three line-disjoint paths for every self-energy contribution, in a way compatible with constructive bounds. On top of that analysis, another one which is scale-dependent is performed: after reduction of some maximal subsets provided by the scale analysis, two vertexdisjoint paths are selected in every self-energy contribution. This construction 
allows to improve the power counting for two point subgraphs, exploiting the particle-hole symmetry of the theory at half-filling, and leads to the desired analyticity result.

Finally an upper bound for the self energy second derivative is combined with a lower bound for the explicit leading self energy Feynman graph [58]. This completes the proof that the Hubbard model violates Salmhofer's criterion, hence is not a Fermi liquid, in contrast with the jellium two dimensional model. More precisely the following theorem summarizes the results of [56, 57, 58.

Theorem 2.2 The radius of convergence of the Hubbard model perturbative series at half-filling is at least $c /|\log T|$, where $T$ is the temperature and c some numerical constant. As $T$ and $\lambda$ jointly tend to 0 in this domain, the self-energy of the model does not display the properties of a Fermi liquid in the sense of Salmhofer, since the second derivative is not uniformly bounded.

We would like now to enter into more technical detail, without drowning the reader. Hence we shall limit ourselves here to the non-perturbative analysis of connected functions in a single RG scale, which is the core mathematical problem. We compare the various models $J_{2}, J_{3}$ and $H_{2}$ to the toy model of section 1.16, and explain why sector analysis plus momentum conservation is suited to analyze the two-dimensional models but fails in three dimensions.

\subsection{D Jellium Model: Why Sectors Work}

We claim that the $J_{2}$ model in a slice is roughly similar to the Toy Model, with dimension $d=3$, provided the momentum slice is divided into angular subpieces called sectors, which play the role of colors.

The naive estimate on the slice propagator is (using integration by parts)

$$
\left|C_{j}(x, y)\right| \leq M^{-j} e^{-\left[M^{-j}|x-y|\right]^{1 / 2}}
$$

(using Gevrey cutoffs $f_{j}$ to get fractional exponential decay). The prefactor $M^{-j}$ corresponds to the volume of integration of the slice, $M^{-2 j}$, divided by the slice estimate of the denominator, $M^{-j}$. This is much worse than the factor $M^{-3 j / 2}$ that would be needed.

But the situation improves if we cut the Fermi slice into smaller pieces (called sectors). Suppose we divide the $j$-th slice into $M^{j}$ sectors, each of size roughly $M^{-j}$ in all three directions.

A sector propagator $C^{j, a}$ has now prefactor $M^{-2 j}$ corresponding to the volume of integration of the sector $M^{-3 j}$ divided by the slice estimate of the denominator, $M^{-j}$. Using integration by parts and Gevrey cutoffs $f_{j a}$ for fractional power decay we get without too much effort the bound

$$
\left|C_{j, a b}(x, y)\right| \leq \delta_{a b} M^{-2 j} e^{-\left[M^{-j}|x-y|\right]^{1 / 2}} .
$$

But since $N=M^{j}$

$$
M^{-2 j}=\frac{M^{-3 j / 2}}{\sqrt{N}}
$$


so that this bound is identical to that of the toy model.

It remains just to explain why the interaction of the model is approximatley of the vector type. This is because of the momentum conservation rule at every vertex.

In two dimensions a rhombus (i.e; a closed quadrilateral whose four sides have equal lengths) is a parallelogram. Hence an approximate rhombus should be an approximate parallelogram.

Momentum conservation $\delta\left(p_{1}+p_{2}+p_{3}+p_{4}\right)$ at each vertex follows from translation invariance of $J_{2}$. Hence $p_{1}, p_{2}, p_{3}, p_{4}$ form a quadrilateral. For $j$ large we have $\left|p_{k}\right| \simeq \sqrt{2 M \mu}$, hence the quadrilateral is an approximate rhombus. Hence the four sectors to which $p_{1}, p_{2}, p_{3}$ and $p_{4}$ should be roughly equal two by two (parallelogram condition).

It means that the interaction is roughly of the color (or Gross-Neveu) type with respect to these angular sectors:

$$
\left(\sum_{a} \bar{\psi}_{a} \psi_{a}\right)\left(\sum_{b} \bar{\psi}_{b} \psi_{b}\right)
$$

In fact this "rhombus rule" is not fully correct for almost degenerate rhombuses. The correct statement is

Lemma 2.3 Fix $m \in \mathbf{Z}^{3}$. The number of 4-tuples $\left\{S_{1}, \cdots S_{4}\right\}$ of sectors for which there exist $k_{i} \in \mathbb{R}^{2}, i=1, \cdots, 4$ satisfying

$$
k_{i}^{\prime} \in S_{i}, \quad\left|k_{i}-k_{i}^{\prime}\right| \leq \mathrm{const} M^{-j}, \quad i=1, \cdots, 4
$$

and

$$
\left|k_{1}+\cdots+k_{4}\right| \leq \operatorname{const}(1+|m|) M^{-j}
$$

is bounded by

$$
\text { const }(1+|m|)^{2} M^{2 j}\{1+j\} .
$$

The $1+j$ factor is special to dimension 2 and is the source of painful technical complications which were developed by Feldman, Magnen, Trubowitz and myself.

The solution uses in fact $M^{j / 2}$ anisotropic angular sectors, which are longer in the tangential direction (of length $M^{-j / 2}$ ). The corresponding propagators still have dual spatial decay because the sectors are still aprroximately flat.

Ultimately the conclusion is unchanged: the radius of convergence of $J_{2}$ in a slice is independent of the slice index $j$.

\subsection{Why Sectors Fail in $d=3$}

In 3D sectors and Gram's bound fail by a full power per vertex!

There is indeed no rhombus rule in $d=3$. A closed quadrilateral with equal sides is not a parallelogram because it can be non-planar; hence it is obtained by rotating half of a planar parallelogram around the diagonal by an arbitrary twisting angle. Therefore the jellium model interaction is not of the vector type. More precisely the analog of Lemma 2.3 is, with similar notations 
Lemma 2.4 The number of 4-tuples $\left\{S_{1}, \cdots S_{4}\right\}$ of sectors for which there exist $k_{i} \in \mathbb{R}^{3}, i=1, \cdots, 4$ satisfying

$$
k_{i}^{\prime} \in S_{i}, \quad\left|k_{i}-k_{i}^{\prime}\right| \leq \mathrm{const} M^{-j}, \quad i=1, \cdots, 4
$$

and

$$
\left|k_{1}+\cdots+k_{4}\right| \leq \operatorname{const}(1+|m|) M^{-j}
$$

is bounded by

$$
\text { const }(1+|m|)^{3} M^{5 j} \text {. }
$$

Remark the absence of the $\log$ factor that was present in $d=2$. But for $d=3$ we find $M^{5 j}$ 4-tuples which correspond to the choice of two sectors $\left(M^{2 j} \times M^{2 j}\right)$ and of one angular twist $M^{j}$. The power counting corresponds to $M^{-3 j}$ per sector propagator. Two propagators pay for one vertex integration $\left(M^{4 j}\right)$ and one sector choice $\left(M^{2 j}\right)$ but there is nothing to pay for the angular twist. Going to anisotropic sectors is possible but there remains still in this case a $M^{j} / 2$ twist factor. After many years of effort, we concluded that the sector method and Gram bound apparently cannot be improved to do better. Hence although $J_{3}$ is expected to be a Fermi liquid in the sense of Salmhofer, new methods have to be developed to treat it constructively [66, 67, 68].

\subsection{The Hadamard method in $x$-space}

The idea of using Hadamard's inequality in $x$-space to overcome the constructive power counting problem of Fermions in three dimensions took Jacques Magnen and myself four years of continuous hard work, with dozens and dozens of various failed trials, from 1991 to 1995 [66]. Another four or five years took place to fine-tune this idea for the multidimensional case with M. Disertori [67]. Another ten years have passed to find a momentum-conserving version, which should at last allow for the proof of Salmhofer's criterion in the three dimensional jellium model 68.

Let us describe the main idea in an informal way.

We remember that the naive estimate (2.4) is far from sufficient for correct power counting. But we also know from perturbative power counting in momentum space that the theory should be just renormalizable. Therefore the $j$-slice propagator $C^{j}$ should behave more as the one of an infrared $\phi_{4}^{4}$ theory, hence should be bounded by

$$
K M^{-2 j} e^{-\left[M^{-j}|x-y|\right]^{1 / 2}} .
$$

In fact this is not totally correct, because it can be shown that the $j$-th slice propagator at almost coinciding points, hence at $|x-y| \simeq 0$ is not bounded by $M^{-2 j}$. Still 2.14 is correct at typical distances for the $J_{3}$ propagator in the $j$-th slice. We know from eg (2.4) that these typical space-time distances should be $|x-y| \simeq M^{j}$. 
Indeed integrating over angles on the Fermi sphere leads to an additional $1 /|x-y|$ decay, because

$$
\int_{0}^{\pi} \sin \theta d \theta d \phi e^{i \cos \theta|x-y|}=\sin |x-y| /|x-y|
$$

Hence for typical distances $|x-y| \simeq M^{j}$ the propagator indeed obeys the improved estimate

$$
\left|C_{j}(x, y)_{|x-y| \simeq M^{j}}\right| \leq K M^{-2 j} e^{-\left[M^{-j}|x-y|\right]^{1 / 2}} .
$$

The problem is that this bound is wrong at small distances. However at small distances there is a bonus, namely the corresponding integration volumes are smaller. Another related problem is that if we use the Gram bound (1.88) to bound a determinant with all the matrix elements $C_{j}\left(x_{p}-y_{q}\right)$ corresponding to large distances $\left|x_{p}-y_{q}\right|$, we still loose the improvement (2.16), because the $L^{2}$ norms in 1.88 will correspond again to propagators at coinciding points!

The solution is to use the Hadamard bounds $1.89-1.90$ because they conserve the decay of the typical propagators.

Remember however, as seen conveniently in (1.91) that Hadamard bounds consume the $1 / n$ ! symmetry factor for $n$ vertices. Hence we can no longer use the explicit tree formula and the method of 46, 47.

But we can still use the standard old-fashioned cluster expansion between cubes. Summarizing:

- Just renormalizable power counting is recovered for the main part of the theory if we use Hadamard's bound rather than Gram's bound.

- The factor $n$ ! is lost in the Hadamard bound at order $n$; this forces us to rely on the on-canonical tool of cluster expansion between cubes

Still, this solves the constructive problem only for the main part of the propagator, the one at typical distances $|x-y| \simeq M^{j}$. However at smaller distances there is a bonus, namely the volume factors for spatial integration are also smaller.

It turns out that the problem of smaller than typical distances can be solved with an auxiliary superrenormalizable decomposition

$$
C^{j}=\sum_{k=0}^{j} C^{j k}
$$

of the propagator. Roughly speaking $C^{j, k}$ corresponds to $|x-y| \simeq M^{k}$, It means that even the single slice theory in $d \geq 3$ is a non-trivial theory that contains a rather non-trivial renormalization, like the one of $\phi_{3}^{4}$. This renormalization can be analyzed by means of the auxiliary scales. The solution is in fact more complicated that what we sketch here, and has to take into account the anisotropy between the space and the imaginary time variables [66, 67]. 
The use of non-canonical lattices of cubes in this method is the signal that we have probably still not found the optimal constructive treatment of $J_{3}$. Recently we found a better decomposition that should allow to check Salmhofer's criterion for $J 3[68$. However in its present stage it will still use a non-canonical cluster expansion between cubes. It would be interesting to find a solution such as the loop vertex expansion that solves the constructive problem in a truly canonical way.

\subsection{D Hubbard Model}

The Hubbard model lives on the square lattice $\mathbb{Z}^{2}$, so that the three dimensional vector $x=\left(x_{0}, \mathbf{x}\right)$ is such that $\mathbf{x}=\left(n_{1}, n_{2}\right) \in \mathbb{Z}^{2}$. From now on we write $v_{1}$ and $v_{2}$ for the two components of a vector $\vec{v}$ along the two axis of the lattice.

At half-filling and finite temperature $T$, the Fourier transform of the propagator of the Hubbard model is:

$$
\hat{C}_{a b}(k)=\delta_{a b} \frac{1}{i k_{0}-e(\mathbf{k})}, \quad e(\mathbf{k})=\cos k_{1}+\cos k_{2},
$$

where $a, b \in\{\uparrow, \downarrow\}$ are the spin indices. The vector $\mathbf{k}$ lives on the two-dimensional torus $\mathbb{R}^{2} /(2 \pi \mathbb{Z})^{2}$. Hence the real space propagator is

$$
C_{a b}(x)=\frac{1}{(2 \pi)^{2} \beta} \sum_{k_{0}} \int_{-\pi}^{\pi} d k_{1} \int_{-\pi}^{\pi} d k_{2} e^{i k x} \hat{C}_{a b}(k) .
$$

Recall that $\left|k_{0}\right| \geq \pi / \beta \neq 0$ hence the denominator in $C(k)$ again can never be 0 at non zero temperature. This is why the temperature provides a natural infrared cut-off. When $T \rightarrow 0$ (which means $\beta \rightarrow \infty$ ) $k_{0}$ becomes a continuous variable, the discrete sum becomes an integral, and the corresponding propagator $C_{0}(x)$ becomes singular on the Fermi surface defined by $k_{0}=0$ and $e(\mathbf{k})=0$. This Fermi surface is a square of side size $\sqrt{2} \pi$ (in the first Brillouin zone) joining the corners $( \pm \pi, 0),(0, \pm \pi)$. We call this square the Fermi square, its corners and faces are called the Fermi faces and corners. Considering the

periodic boundary conditions, there are really four Fermi faces, but only two Fermi corners.

In the following to simplify notations we will write:

$$
\int d^{3} k \equiv \frac{1}{\beta} \sum_{k_{0}} \int d^{2} k \quad, \quad \int d^{3} x \equiv \frac{1}{2} \int_{-\beta}^{\beta} d x_{0} \sum_{\mathbf{x} \in \mathbb{Z}^{2}} .
$$

In determining the spatial decay we recall that by anti-periodicity

$$
C(x)=f\left(x_{0}, \mathbf{x}\right):=\sum_{m \in \mathbb{Z}}(-1)^{m} C_{0}\left(x_{0}+\frac{m}{T}, \mathbf{x}\right) .
$$

where $C_{0}$ is the propagator at $T=0$. Indeed the function $f$ is anti-periodic and its Fourier transform is the right one. 
The interaction of the Hubbard model is again 2.3):

$$
S_{V}=\lambda \int_{V} d^{3} x\left(\sum_{a} \bar{\psi} \psi\right)^{2}(x)
$$

where $V:=[-\beta, \beta] \times V^{\prime}$ and $V^{\prime}$ is an auxiliary finite volume cutoff in two dimensional space that will be sent later to infinity.

\subsection{Scale Analysis}

The theory has a natural lattice spatial cutoff. To implement the renormalization group analysis, we introduce as usually a compact support function $u(r) \in \mathcal{C}_{0}^{\infty}(\mathrm{R})$ (it is convenient to choose it to be Gevrey of order $\alpha<1$ so as to ensure fractional exponential decrease in the dual space) which satisfies:

$$
u(r)=0 \quad \text { for }|r|>2 ; u(r)=1 \quad \text { for }|r|<1 .
$$

With this function, given a constant $M \geq 2$, we can construct a partition of unity

$$
\begin{aligned}
1 & =\sum_{i=0}^{\infty} u_{i}(r) \forall r \neq 0 ; \\
u_{0}(r) & =1-u(r) ; u_{i}(r)=u\left(M^{2(i-1)} r\right)-u\left(M^{2 i} r\right) \text { for } i \geq 1
\end{aligned}
$$

The propagator is then divided into slices according to this partition

$$
C(k)=\sum_{i=0}^{\infty} C_{i}(k)
$$

where

$$
C_{i}(k)=C(k) u_{i}\left[k_{0}^{2}+e^{2}(\mathbf{k})\right] .
$$

(indeed $k_{0}^{2}+e^{2}(\mathbf{k}) \geq T^{2}>0$ ).

In a slice of index $i$ the cutoffs ensure that the size of $k_{0}^{2}+e^{2}(\mathbf{k})$ is roughly $M^{-2 i}$. More precisely in the slice $i$ we must have

$$
M^{-2 i} \leq k_{0}^{2}+e^{2}(\mathbf{k}) \leq 2 M^{2} M^{-2 i}
$$

The corresponding domain is a three dimensional volume whose section through the $k_{0}=0$ plane is the shaded region pictured in Figure 6 .

Remark that at finite temperature, the propagator $C_{i}$ vanishes for $i \geq$ $i_{\max }(T)$ where $M^{i_{\max }(T)} \simeq 1 / T\left(\right.$ more precisely $i_{\max }(T)=E\left(\log \frac{M \sqrt{2}}{\pi T} / \log M\right)$, where $E$ is the integer part), so there is only a finite number of steps in the renormalization group analysis.

Let us state first a simple result, for a theory whose propagator is only $C_{i}$, hence corresponding to a generic step of the renormalization group: 


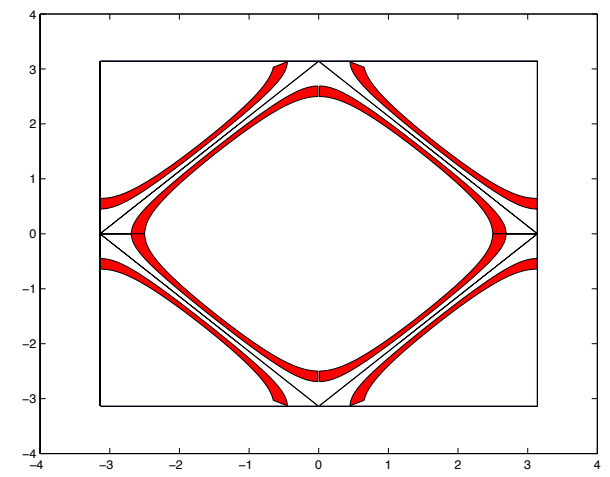

Figure 6: A single slice of the renormalization group

Theorem 1 The Schwinger functions of the theory with propagator $C_{i}$ and interaction (2.22) are analytic in $\lambda$ in a disk of radius $R_{i}$ which is at least $c / i$ for a suitable constant $c$ :

$$
R_{i} \geq c / i
$$

The rest of this section is devoted to the definitions and properties of $H_{2}$ sectors, their scaled decay and momentum conservation rules. As discussed already this result is a first step towards the rigorous proof [57, 58] that $H_{2}$ is not a Fermi liquid in the sense of Salmhofer.

\subsection{Sectors}

This section is extracted from [56].

The "angular" analysis is completely different from the jellium case. We remark first that in a slice, $k_{0}^{2}+e^{2}(\mathbf{k})$ is of order $M^{-2 i}$, but this does not fix the size of $e^{2}(\mathbf{k})$ itself, which can be of order $M^{-2 j}$ for some $j \geq i$. In order for sectors defined in momentum space to correspond to propagators with dual decay in direct space, it is essential that their length in the tangential direction is not too big, otherwise the curvature is too strong for stationary phase methods to apply. This was discussed first in 62. This leads us to study the curve $\left(\cos k_{1}+\cos k_{2}\right)^{2}=M^{-2 j}$ for arbitrary $j \geq i$. We can by symmetry restrict ourselves to the region $0 \leq k_{1} \leq \pi / 2, k_{2}>0$. It is then easy to compute the curvature radius of that curve, which is

$$
R=\frac{\left(\sin ^{2} k_{1}+\sin ^{2} k_{2}\right)^{3 / 2}}{\left|\cos k_{1} \sin ^{2} k_{2}+\cos k_{2} \sin ^{2} k_{1}\right|} .
$$

We can also compute the distance $d\left(k_{1}\right)$ to the critical curve $\cos k_{1}+\cos k_{2}=0$, and the width $w\left(k_{1}\right)$ of the band $M^{-j} \leq\left|\cos k_{1}+\cos k_{2}\right| \leq \sqrt{2} M \cdot M^{-j}$. We can 
then easily check that

$$
\begin{gathered}
d\left(k_{1}\right) \simeq w\left(k_{1}\right) \simeq \frac{M^{-j}}{M^{-j / 2}+k_{1}}, \\
R\left(k_{1}\right) \simeq \frac{k_{1}^{3}+M^{-3 j / 2}}{M^{-j}},
\end{gathered}
$$

where $f \simeq g$ means that on the range $0 \leq k_{1} \leq \pi / 2$ we have inequalities $c f \leq g \leq d f$ for some constants $c$ and $d$.

Defining the anisotropic length

$$
l\left(k_{1}\right)=\sqrt{w\left(k_{1}\right) R\left(k_{1}\right)} \simeq M^{-j / 2}+k_{1},
$$

the condition in 62 is that the sector length should not be bigger than that anisotropic length. This leads to the idea that $k_{1}$ or an equivalent quantity should be sliced according to a geometric progression from 1 to $M^{-j / 2}$ to form the angular sectors in this model.

For symmetry reasons it is convenient to introduce a new orthogonal but not normal basis in momentum space $\left(e_{+}, e_{-}\right)$, defined by $e_{+}=(1 / 2)(\pi, \pi)$ and $e_{-}=(1 / 2)(-\pi, \pi)$. Indeed if we call $\left(k_{+}, k_{-}\right)$the coordinates of a momentum $k$ in this basis, the Fermi surface is given by the simple equations $k_{+}= \pm 1$ or $k_{-}= \pm 1$. This immediately follows from the identity

$$
\cos k_{1}+\cos k_{2}=2 \cos \left(\pi k_{+} / 2\right) \cos \left(\pi k_{-} / 2\right) .
$$

(Note however that the periodic b.c. are more complicated in that new basis). Instead of slicing $e(\mathbf{k})$ and $k_{1}$, it is then more symmetric to slice directly $\cos \left(\pi k_{+} / 2\right)$ and $\cos \left(\pi k_{-} / 2\right)$.

Guided by these considerations we introduce the partition of unity

$$
1=\sum_{s=0}^{i} v_{s}(r) ;\left\{\begin{array}{l}
v_{0}(r)=1-u\left(M^{2} r\right) \\
v_{s}=u_{s+1} \\
v_{i}(r)=u\left(M^{2 i} r\right)
\end{array} \quad \text { for } 1 \leq s \leq i-1\right.
$$

and define

$$
C_{i}(k)=\sum_{\sigma=\left(s_{+}, s_{-}\right)} C_{i, \sigma}(k)
$$

where

$$
\left.C_{i, \sigma}(k)=C_{i}(k) v_{s_{+}}\left[\cos ^{2}\left(\pi k_{+} / 2\right)\right] v_{s_{-}}\left[\cos ^{2} \pi k_{-} / 2\right)\right] .
$$

We remark that using (2.27) in order for $C_{i, \sigma}$ not to be 0 , we need to have $s_{+}+s_{-} \geq i-2$. We define the "depth" $l(\sigma)$ of a sector to be $l=s_{+}+s_{-}-i+2$.

To get a better intuitive picture of the sectors, we remark that they can be classified into different categories:

- the sectors $(0, \mathrm{i})$ and $(\mathrm{i}, 0)$ are called the middle-face sectors

- the sectors (s,i) and (i,s) with $0<s<i$ are called the face sectors 
- the sector $(\mathrm{i}, \mathrm{i})$ is called the corner sector

- the sectors $(\mathrm{s}, \mathrm{s})$ with $(i-2) / 2 \leq s<i$ are called the diagonal sectors

- the others are the general sectors

Finally the general or diagonal sectors of depth 0 for which $s_{+}+s_{-}=i-2$ are called border sectors.

If we consider the projection onto the $\left(k_{+}, k_{-}\right)$plane, taking into account the periodic b.c. of the Brillouin zone, the general and diagonal sectors have 8 connected components, the face sectors have 4 connected components, the middle face sectors and the corner sector have 2 connected components. In the three dimensional space-time, if we neglect the discretization of the Matsubara frequencies, these numbers would double except for the border sectors.

\subsection{Scaled decay}

Lemma 2.5 Using Gevrey cutoffs of degree $\alpha<1$, the propagator $C_{i, \sigma}$ obeys the scaled decay

$$
\left|C_{i, \sigma}\right| \leq c . M^{-i-l} e^{-\left[d_{i, \sigma}(x, y)\right]^{\alpha}}
$$

where

$$
d_{i, \sigma}(x, y)=\left\{M^{-i}\left|x_{0}-y_{0}\right|+M^{-s_{+}}\left|x_{+}-y_{+}\right|+M^{-s_{-}}\left|x_{-}-y_{-}\right|\right\} .
$$

Proof This is essentially Fourier analysis and integration by parts. If $x=$ $\left(n_{1}, n_{2}\right) \in \mathbb{Z}^{2}$, we define $\left(x_{+}, x_{-}\right)=(\pi / 2)\left(n_{1}+n_{2}, n_{2}-n_{1}\right)$. The vector $\left(x_{+}, x_{-}\right)$ then belongs to $(\pi / 2) \mathbb{Z}^{2}$ but with the additional condition that $x_{+}$and $x_{-}$have the same parity.

Defining, for $X \in[(\pi / 2) \mathbb{Z}]^{2}$

$$
\begin{aligned}
D_{i, \sigma}(X)= & (1 / 2) \frac{1}{8 \beta} \sum_{k_{0}} \int_{-2}^{+2} d k_{+} \int_{-2}^{+2} d k_{-} e^{i\left(k_{0} x_{0}+k_{+} x_{+}+k_{-} x_{-}\right)} \\
& \frac{u_{i}\left[k_{0}^{2}+4 \cos ^{2}\left(\pi k_{+} / 2\right) \cos ^{2}\left(\pi k_{-} / 2\right)\right]}{i k_{0}-2 \cos \left(\pi k_{+} / 2\right) \cos \left(\pi k_{-} / 2\right)} \\
& v_{s_{+}}\left[\cos ^{2}\left(\pi k_{+} / 2\right)\right] v_{s_{-}}\left[\cos ^{2}\left(\pi k_{-} / 2\right)\right]
\end{aligned}
$$

we note that $C_{i, \sigma}(X)=D_{i, \sigma}(X)$ for $X$ satisfying the parity condition.

(Remember the Jacobian $\frac{\pi^{2}}{2}$ from $d k_{1} d k_{2}$ to $d k_{+} d k_{-}$, and the initial domain of integration that is doubled.)

The volume of integration trivially gives a factor $M^{-i}$ for the $k_{0}$ sum and factors $M^{-s_{+}}$and $M^{-s_{-}}$for the $k_{+}$and $k_{-}$integration (see 2.44 below). The integrand is trivially bounded by $M^{i}$ on the integration domain, and this explains the prefactor $c M^{-i-l}$ in 2.37). 
We then apply standard integration by parts techniques to formulate the decay. From e.g. Lemma 10 in 54 we know that to obtain the scaled decay of Lemma 2.5 we have only to check the usual derivative bounds in Fourier space:

$$
\left\|\frac{\partial^{n_{0}}}{\partial k_{0}^{n_{0}}} \frac{\partial^{n_{+}}}{\partial k_{+}^{n_{+}}} \frac{\partial^{n_{-}}}{\partial k_{0}^{n_{-}}} \hat{D}_{i, \sigma}\right\| \leq A \cdot B^{n} M^{i n_{0}} M^{s_{+} n_{+}} M^{s_{-} n_{-}}(n !)^{1 / \alpha}
$$

where $n=n_{0}+n_{+}+n_{-}$, and the derivative $\frac{\partial}{\partial k_{0}}$ really means the natural finite difference operator $(1 / 2 \pi T)\left(f\left(k_{0}+2 \pi T\right)-f\left(k_{0}\right)\right)$ acting on the discrete Matsubara frequencies. The norm is the ordinary sup norm.

But from 2.39,

$$
\begin{aligned}
\hat{D}_{i, \sigma}(k)= & \frac{1}{16 \beta} \frac{u_{i}\left[k_{0}^{2}+4 \cos ^{2}\left(\pi k_{+} / 2\right) \cos ^{2}\left(\pi k_{-} / 2\right)\right]}{i k_{0}-2 \cos \left(\pi k_{+} / 2\right) \cos \left(\pi k_{-} / 2\right)} \\
& v_{s_{+}}\left[\cos ^{2}\left(\pi k_{+} / 2\right)\right] v_{s_{-}}\left[\cos ^{2}\left(\pi k_{-} / 2\right)\right]
\end{aligned}
$$

and the derivatives are bounded easily using the standard rules for derivation, product and composition of Gevrey functions, or by hand, using the support properties of the $v_{s_{+}}$and $v_{s_{-}}$fonctions. For instance a derivative $\frac{\partial}{\partial k_{+}}$can act on the $v_{s_{+}}\left[\cos ^{2}\left(\pi k_{+} / 2\right)\right]$ factor, in which case it is easily directly bounded by $c M^{s_{+}}$ for some constant $c$. When it acts on $u_{i}\left[k_{0}^{2}+4 \cos ^{2}\left(\pi k_{+} / 2\right) \cos ^{2}\left(\pi k_{-} / 2\right)\right]$ it is easily bounded by $c \cdot M^{2 i-s_{+}-2 s_{-}}$hence by $c \cdot M^{s_{+}}$, using the relation $s_{+}+s_{-} \geq$ $i-2$. When it acts on the denominator $\left[i k_{0}-2 \cos \left(\pi k_{+} / 2\right) \cos \left(\pi k_{-} / 2\right)\right]^{-1}$. it is bounded by $c . M^{i-s_{-}}$, hence again by $c . M^{s_{+}}$, using the relation $s_{+}+s_{-} \geq i-2$. Finally when it acts on a $\cos \left(\pi k_{+} / 2\right)$ created by previous derivations, it costs directly $c . M^{s_{+}}$. The factorial factor $(n !)^{1 / \alpha}$ in 2.40 comes naturally from deriving the cutoffs, which are Gevrey functions of order $\alpha$; deriving other factors give smaller factorials (with power 1 instead of $1 / \alpha$ ).

Finally a last remark: to obtain the Lemma for the last slice, $i=i_{\max }(T)$, one has to take into account the fact that $x_{0}$ lies in a compact circle, so that there is really no long-distance decay to prove.

\subsection{Support Properties}

If $C_{i, \sigma}(k) \neq 0$, the momentum $k$ must obey the following bounds:

$$
\begin{gathered}
\quad\left|k_{0}\right| \leq \sqrt{2} M M^{-i} \\
\begin{cases}M^{-1} \leq\left|\cos \left(\pi k_{ \pm} / 2\right)\right| \leq 1 & \text { for } s_{ \pm}=0 \\
M^{-s_{ \pm}-1} \leq\left|\cos \left(\pi k_{ \pm} / 2\right)\right| \leq \sqrt{2} M^{-s_{ \pm}} & \text {for } 1 \leq s_{ \pm} \leq i-1, \\
\left|\cos \left(\pi k_{ \pm} / 2\right)\right| \leq \sqrt{2} M^{-i} & \text { for } s_{ \pm}=i\end{cases}
\end{gathered}
$$

In the support of our slice in the first Brillouin zone we have $\left|k_{+}\right|<2$ and $\left|k_{-}\right|<2$ (this is not essential but the inequalities are strict because $i \geq 1$ ). It is convenient to associate to any such component $k_{ \pm}$a kind of "fractional part" 
called $q_{ \pm}$defined by $q_{ \pm}=k_{ \pm}-1$ if $k_{ \pm} \geq 0$ and $q_{ \pm}=k_{ \pm}+1$ if $k_{ \pm}<0$, so that $0 \leq\left|q_{ \pm}\right| \leq 1$. Then the bounds translate into

$$
\begin{cases}2 / \pi M \leq\left|q_{ \pm}\right| \leq 1 & \text { for } s_{ \pm}=0 \\ 2 M^{-s_{ \pm}} / \pi M \leq\left|q_{ \pm}\right| \leq \sqrt{2} M^{-s_{ \pm}} & \text {for } 1 \leq s_{ \pm} \leq i-1 \\ \left|q_{ \pm}\right| \leq \sqrt{2} M^{-i} & \text { for } s_{ \pm}=i\end{cases}
$$

\subsection{Momentum conservation rules at a vertex}

Let us consider that the four momenta $k_{1}, k_{2}, k_{3}, k_{4}$, arriving at a given vertex $v$ belong to the support of the four sectors $\sigma_{1}, \sigma_{2}, \sigma_{3}, \sigma_{4}$, in slices $i_{1}, i_{2}, i_{3}, i_{4}$. In Fourier space the vertex 2.22 implies constraints on the momenta. Each spatial component of the sum of the four momenta must be an integer multiple of $2 \pi$ in the initial basis, and the sum of the four Matsubara frequencies must also be zero.

In our tilted basis $\left(e_{+}, e_{-}\right)$, this translates into the conditions:

$$
\begin{aligned}
k_{1,0}+k_{2,0}+k_{3,0}+k_{4,0} & =0, \\
k_{1,+}+k_{2,+}+k_{3,+}+k_{4,+} & =2 n_{+}, \\
k_{1,-}+k_{2,-}+k_{3,-}+k_{4,-} & =2 n_{-},
\end{aligned}
$$

where $n_{+}$and $n_{-}$must have identical parity.

We want to rewrite the two last equations in terms of the fractional parts $q_{1}, q_{2}, q_{3}$ and $q_{4}$.

Since an even sum of integers which are \pm 1 is even, we find that (2.46) and 2.47) imply

$$
\begin{aligned}
& q_{1,+}+q_{2,+}+q_{3,+}+q_{4,+}=2 m_{+}, \\
& q_{1,-}+q_{2,-}+q_{3,-}+q_{4,-}=2 m_{-},
\end{aligned}
$$

with $m_{+}$and $m_{-}$integers. Let us prove now that except in very special cases, these integers must be 0 . Since $\left|q_{j, \pm}\right| \leq 1,\left|m_{ \pm}\right| \leq 2$. But $\left|q_{j, \pm}\right|=1$ is possible only for $s_{j, \pm}=0$. Therefore $\left|m_{ \pm}\right|=2$ implies $s_{j, \pm}=0 \forall j$. Now suppose e.g. $\left|m_{+}\right|=1$. Then $s_{j,+}$ is 0 for at least two values of $j$. Indeed for $s_{j, \pm} \neq 0$ we have $\left|q_{j, \pm}\right| \leq \sqrt{2} M^{-1}$, and assuming $3 \sqrt{2} M^{-1}<1$, equation 2.48) could not hold.

We have therefore proved

Lemma $2.6 m_{+}=0$ unless $s_{j,+}$ is 0 for at least two values of $j$, and $m_{-}=0$ unless $s_{j,-}$ is 0 for at least two values of $j$.

Let us analyze in more detail equations (2.48) and 2.49 for $\left|m_{+}\right|=\left|m_{-}\right|=$ 0 . Consider e.g. 2.48). By a relabeling we can assume without loss of generality that $s_{1,+} \leq s_{2,+} \leq s_{3,+} \leq s_{4,+}$ Then either $s_{1,+}=i_{1}$ or $s_{1,+}<i_{1}$, in which case combining equations 2.48) and 2.44 we must have:

$$
3 \sqrt{2} M^{-s_{2,+}} \geq 2 M^{-s_{1,+}} / \pi M
$$


which means

$$
s_{2,+} \leq s_{1,+}+1+\frac{\log (3 \pi / \sqrt{2})}{\log M}
$$

This implies

$$
\left|s_{2,+}-s_{1,+}\right| \leq 1
$$

if $M>3 \pi / \sqrt{2}$, which we assume from now on.

The conclusion is:

Lemma 2.7 If $m_{ \pm}=0$, either the smallest index $s_{1, \pm}$ coincides with its scale $i_{1}$, or the two smallest indices among $s_{j, \pm}$ differ by at most one unit.

Now we can summarize the content of both Lemmas in a slightly weaker but simpler lemma:

Lemma 2.8

A) (single slice case)

The two smallest indices among $s_{j,+}$ for $j=1,2,3,4$ differ by at most one unit, and the two smallest indices among $s_{j,-}$ for $j=1,2,3,4$ differ by at most one unit.

\section{B) Multislice case}

The two smallest indices among $s_{j,+}$ for $j=1,2,3,4$ differ by at most one unit or the smallest one, say $s_{1,+}$ must coincide with its scale $i_{1}$, which must then be strictly smaller than the three other scales $i_{2}, i_{3}$ and $i_{4}$. Exactly the same statement holds independently for the minus direction.

This lemma allows to check that again the single scale analysis works and leads to a radius of convergence independent of the slice 56.

\subsection{Multiscale Analysis}

The half-filling point is very convenient since the particle-hole exact symmetry at this point ensures that there is no flow for the Fermi surface itself.

Using the sector decomposition and the momentum conservation we find that power counting of the 2D Hubbard model is essentially similar to one dimensional case with logarithmic corrections [57. Expanding the two point function to second order we can even find lower bounds which prove that this model is not a Fermi liquid in the sense of Salmhofer [58.

\subsection{Acknowledgments}

I thank J. Magnen, M. Disertori, M. Smerlak and L. Gouba for contributing various aspects of this work. 


\section{References}

[1] J. Glimm and A. Jaffe, Quantum Physics. A functional integral point of view. Mc Graw and Hill, New York, 1981.

[2] R. Feynman and A. Hibbs, Quantum Mechanics and Path Integrals, Mc Graw and Hill, New York 1965.

[3] M. Peskin and Daniel V. Schroeder (Contributor), An Introduction to Quantum Field Theory, Perseus Publishing, (1995).

[4] C. Itzykson and J.-B. Zuber, Quantum Field Theory, McGraw Hill, 1980.

[5] P. Ramond, Field Theory, Addison-Wesley, 1994.

[6] J. Zinn-Justin, Quantum Field Theory and Critical Phenomena, Oxford University Press, 2002.

[7] C. Itzykson and J.M Drouffe, Statistical Field Theory, Volumes 1 and 2, Cambridge University Press 1991.

[8] Giorgio Parisi, Statistical Field Theory, Perseus Publishing 1998.

[9] V. Rivasseau, From Perturbative to Constructive Renormalization, Princeton University Press, 1991.

[10] J.P. Eckmann, J. Magnen and R. Sénéor, Decay properties and Borel summability for the Schwinger functions in $P(\phi)_{2}$ theories, Comm. Math. Phys. 39, 251 (1975).

[11] J. Magnen and R. Sénéor, Phase space cell expansion and Borel summability for the Euclidean $\phi_{3}^{4}$ theory, Comm Math. Phys. 56, 237 (1977).

[12] A. Sokal, An improvement of Watson's theorem on Borel summability, Journ. Math. Phys. 21, 261 (1980).

[13] F. Bergeron, G. Labelle and P. Leroux, Combinatorial Species and Tree-like Structures, Cambridge University Press (1998).

[14] Constructive Physics, Lecture Notes in Physics 446, Springer Verlag, 1995.

[15] V. Rivasseau, Constructive Field Theory and Applications: Perspectives and Open Problems, Journ. Math. Phys. 41, 3764 (2000).

[16] V. Rivasseau, Constructive Matrix Theory, arXiv:hep-ph/0706.1224, JHEP 09 (2007) 008.

[17] J. Magnen and V. Rivasseau, Constructive field theory without tears, math/ph/0706.2457, Ann. Henri Poincaré 9 403-424 (2008).

[18] R. Gurau, J. Magnen and V. Rivasseau, Tree Quantum Field Theory, arXiv:0807.4122, Annales Henri Poincaré, Vol 10 no 5, (2009). 
[19] V. Rivasseau, Constructive Field Theory in Zero Dimension, arXiv:0906.3524, Advances in Mathematical Physics, Volume 2009 (2009), Article ID 180159.

[20] V. Rivasseau and Zhituo Wang, How are Feynman graphs resummed by the Loop Vertex Expansion, arXiv 1006...

[21] T. Krajewski, V. Rivasseau, A. Tanasa and Zhituo Wang, Topological Graph Polynomials and Quantum Field Theory, Part I: Heat Kernel Theories, arXiv:0811.0186, Journ. Noncommut. Geometry 4, (2010) 29182

[22] K. Wilson, Renormalization group and critical phenomena, II Phase space cell analysis of critical behavior, Phys. Rev. B 4, 3184 (1974).

[23] N. Nakanishi, Graph Theory and Feynman integrals, Gordon and Breach, New York 1971.

[24] K. Symanzik, in Local Quantum Theory, R. Jost Ed. (Varenna, 1968) Academic Press, New York (1969), 285.

[25] K. Hepp, Théorie de la renormalisation, Berlin, Springer Verlag, 1969.

[26] N. Bogoliubov and Parasiuk, Acta Math. 97, 227 (1957).

[27] W. Zimmermann, Convergence of Bogoliubov's method for renormalization in momentum space, Comm. Math. Phys. 15, 208 (1969).

[28] J. Polchinski, Nucl. Phys. B 231, 269 (1984).

[29] C. Kopper, Rigorous Polchinski method....

[30] M. Bergère and Y.M.P. Lam, Bogoliubov-Parasiuk theorem in the $\alpha$ parametric representation, Journ. Math. Phys. 17, 1546 (1976).

[31] M. Bergère and J.B. Zuber, Renormalization of Feynman amplitudes and parametric integral representation, Comm. Math. Phys. 35, 113 (1974).

[32] C. de Calan and V. Rivasseau, Local existence of the Borel transform in Euclidean $\phi_{4}^{4}$, Comm. Math. Phys. 82, 69 (1981).

[33] G. 'tHooft and M. Veltman, Nucl. Phys. B50, 318 (1972).

[34] H. Grosse and R. Wulkenhaar, Renormalization of $\phi^{4}$-theory on noncommutative $\mathbb{R}^{4}$ in the matrix base, Commun. Math. Phys. 256 (2005), no. 2, 305-374, hep-th/0401128

[35] H. Grosse and R. Wulkenhaar, The beta-function in duality-covariant noncommutative $\phi^{4}$-theory, Eur. Phys. J. C35 (2004) 277-282, hepth/0402093. 
[36] M. Disertori and V. Rivasseau, Two and three loops Beta fonction of Non commutative Phi44 Theory, arXiv:hep-th/0610224, Eur. Phys. Journ. C 50 (2007), 661.

[37] M. Disertori, R. Gurau, J. Magnen and V. Rivasseau, Vanishing of Beta fonction of Non commutative $\Phi_{4}^{4}$ Theory to all orders, arXiv:hepth/0612251, Physics Letters B, 649, 95-102 (2007).

[38] D. Brydges and T. Kennedy, Mayer expansions and the Hamilton-Jacobi equation, Journal of Statistical Physics, 48, 19 (1987).

[39] A. Abdesselam and V. Rivasseau, Trees, forests and jungles: A botanical garden for cluster expansions, arXiv:hep-th/9409094, in Constructive Physics, Lecture Notes in Physics 446, Springer Verlag, 1995.

[40] V. Rivasseau and Z. Wang, Loop Vertex Expansion for $\phi^{2 k}$ Theory in Zero Dimension, arXiv:1003.1037.

[41] J. Feldman, Renormalization Group and Fermionic Functional Integrals, CRM Monograph Series, Volume 16, published by the AMS.

[42] Tree Matrix

[43] G. Benfatto, G. Gallavotti, Renormalization Group, Princeton University Press, 1995.

[44] Manfred Salmhofer, Renormalization: An Introduction, Texts and Monographs in Physics, Springer Verlag, 1999.

[45] Vieri Mastropietro,

[46] A. Lesniewski, Effective Action for the Yukawa 2 Quantum Field Theory, Commun. Math. Phys. 108, 437 (1987).

[47] A. Abdesselam and V. Rivasseau, Explicit fermionic tree expansions arXiv:cond-mat/9712055, Lett. Math. Phys. Vol. 44, Number 1, 77-88

[48] M. Salmhofer, Commun. Math. Phys.194, 249 (1998).

[49] J. Feldman, H. Knor̈rer and E. Trubowitz, A two dimensional Fermi Liquid, Commun. Math.Phys. 247, 1-319, 2004 and Reviews in Math. Physics, 15, 9, 949-1169, (2003). papers accessible at http://www.math.ubc.ca/ feldman/fl.html.

[50] R. Gurau, J. Magnen, V. Rivasseau and F. Vignes-Tourneret, Renormalization of Non-Commutative $\Phi_{4}^{4}$ Field Theory in $x$ Space, Commun. Math. Phys. 267, 515 (2006).

[51] G. Benfatto and G. Gallavotti Perturbation theory of the Fermi surface in a quantum liquid. A general quasi-particle formalism and one dimensional systems, Journ. Stat. Physics 59, 541, 1990. 
[52] J. Feldman and E. Trubowitz, Perturbation Theory for Many Fermions Systems, Helv. Phys. Acta 63, 156 (1990).

[53] J. Feldman and E. Trubowitz, The Flow of an Electron-Phonon System to the Superconducting State, Helv. Phys. Acta 64, 213 (1991).

[54] M. Disertori and V. Rivasseau, Interacting Fermi liquid in two dimensions at finite temperature, Part I: Convergent Attributions, avec M. Disertori, Commun. Math. Phys. 215, 251 (2000).

[55] M. Disertori and V. Rivasseau, A Rigorous Proof of Fermi Liquid Behavior for Jellium Two-Dimensional Interacting Fermions, Phys. Rev. Lett. 85, 361 (2000).

[56] V. Rivasseau, The two dimensional Hubbard Model at half-filling: I. Convergent Contributions, Journ. Stat. Phys. 106, 693-722; (2002).

[57] S. Afchain, J. Magnen and V. Rivasseau, Renormalization of the 2-point function of the Hubbard Model at half-filling, Ann. Henri Poincaré 6, 399, (2005).

[58] S. Afchain, J. Magnen and V. Rivasseau, The Hubbard Model at half-filling, part III: the lower bound on the self-energy, Ann. Henri Poincaré 6, 449 (2005).

[59] G. Benfatto, A. Giuliani, V. Mastropietro Low temperature analysis of two dimensional Fermi systems with symmetric Fermi surface arXiv:condmat/0207210

[60] G. Benfatto, A. Giuliani, V. Mastropietro, Fermi liquid behavior in the 2D Hubbard model at low temperatures arXiv:cond-mat/0507686

[61] P.W. Anderson, Luttinger liquid behavior of the normal metallic state of the 2D Hubbard model, Phys Rev Lett. 64 1839-1841 (1990).

[62] J. Feldman, J. Magnen, V. Rivasseau and E. Trubowitz, An Infinite Volume Expansion for Many Fermion Green's functions, Helv. Phys. Acta 65, 679 (1992).

[63] J. Feldman, J. Magnen, V. Rivasseau and E. Trubowitz, An Intrinsic 1/N Expansion for Many Fermion Systems, Europhys. Letters 24, 437 (1993).

[64] J. Feldman, J. Magnen, V. Rivasseau and E. Trubowitz, Two dimensional Many Fermion Systems as Vector Models, Europhys. Letters 24, 521 (1993).

[65] M. Salmhofer, Continuous renormalization for fermions and Fermi liquid theory, Comm. Math. Phys. 194, 249 (1998).

[66] J. Magnen and V. Rivasseau, A single scale Infinite Volume Expansion for Three-Dimensional Many Fermion Green's Functions, Mathematical Physics Electronic Journal 1, n3 (1995). 
[67] M. Disertori, J. Magnen and V. Rivasseau, Interacting Fermi liquid in three dimensions at finite temperature: Part I: Convergent Contributions, Annales Henri Poincaré 2, 733-806 (2001).

[68] M. Disertori, J. Magnen and V. Rivasseau, in preparation. 